\title{
Infraestructura y crecimiento económico en el Perú
}

\author{
Roberto Urrunaga y Carlos Aparicio
}

RESUMEN

Se revisa la literatura donde se analiza la importancia de la infraestructura para el crecimiento económico y se efectúa una estimación econométrica a fin de recoger la relación entre ambas variables en el caso peruano. Para ello, se utiliza un panel de datos con información para las 24 regiones del Perú correspondientes al período 1980-2009, bajo distintos estimadores. Los resultados econométricos obtenidos confirman que las infraestructuras de servicios públicos (carreteras, electricidad y telecomunicaciones) resultan relevantes para explicar las diferencias transitorias en el producto regional, de acuerdo con las teorías neoclásicas de crecimiento exógeno. Por otra parte, se encuentra evidencia que respalda la presencia de diferencias significativas en las repercusiones de las distintas infraestructuras en el producto per cápita de cada región. Por consiguiente, las autoridades de política deberían agilizar el desarrollo de proyectos que permitan disminuir las brechas en infraestructura que imponen trabas al desarrollo de las regiones del Perú.

PALABRAS CLAVE

CLASIFICACIÓN JEL

AUTORES
Infraestructura física, carreteras, energía eléctrica, telecomunicaciones, crecimiento económico, desarrollo regional, producto interno bruto, modelos econométricos, Perú

C33, H54, L90, O11, O18, O40, R11

Roberto Urrunaga es profesor e investigador principal del Departamento de Economía y del Centro de Investigación de la Universidad del Pacífico (Lima, Perú).urrunaga_rl@up.edu.pe

Carlos Aparicio es analista del Departamento de Investigación Económica de la Superintendencia de Banca, Seguros y Afp del Perú y profesor del Departamento de Economía de la Universidad del Pacífico (Lima, Perú).caparicio@sbs.gob.pe 


\section{I}

\section{Introducción}

La infraestructura se relaciona directamente con la producción y estimula el crecimiento económico porque se trata de un insumo fundamental para la realización de las actividades privadas. Algunos estudios en que se resumen los principales resultados sobre esta relación son los de Straub (2008a); González, Guasch y Serebrisky (2007), y Cárdenas, Gaviria y Meléndez (2005).

El principal mecanismo por medio del cual la infraestructura afecta al producto y al crecimiento económico se encuentra en la mejora de la productividad del capital, que será más importante cuanto mayor sea la complementariedad entre la infraestructura y la inversión productiva de las empresas. De acuerdo con Straub (2008a), otros mecanismos se hallan en las actividades de mantenimiento de las infraestructuras, las que aumentan al incrementarse su construcción; los costos de ajuste, que se reducen debido a los menores costos logísticos que producen las nuevas inversiones; el mejoramiento de la productividad laboral, al contar los trabajadores con mejores tecnologías de la información y la comunicación (TIC) y mejores condiciones de salud y educación; y la disminución de los costos de transporte, derivada del aprovechamiento de las economías de escala y de ámbito.

El estudio de la relación entre infraestructura y crecimiento productivo resulta particularmente interesante en el caso peruano, debido al gran dinamismo que ha

$\square$ Los autores agradecen la colaboración de Carlos Cubas y Regina Ruiz en el desarrollo de este documento, así como los comentarios realizados a una versión preliminar por un árbitro anónimo de la Revista CEPAL. Cualquier error u omisión es de responsabilidad exclusiva de los autores. venido experimentando su economía y a los importantes avances que ha logrado con respecto al desarrollo de infraestructuras de servicios públicos. En este contexto, la hipótesis principal que se pretende demostrar es que dicha infraestructura repercute marcadamente en el producto y el crecimiento económico de las regiones peruanas. Además, se procura analizar si existen diferencias significativas en la contribución de las infraestructuras al desarrollo productivo de las regiones del Perú. Con este propósito, se emplea un panel de datos que contiene información respecto de las 24 regiones peruanas para el período 1980-2009, bajo distintos estimadores econométricos. Dadas las restricciones de información existentes, se trabaja con información relacionada con las infraestructuras de carreteras, telecomunicaciones y energía eléctrica.

La relevancia de realizar este tipo de estudios radica en que tanto el Perú como muchos otros países latinoamericanos enfrentan todavía problemas de infraestructura que pueden imponer limitaciones a las oportunidades de crecimiento, por lo que se requiere llamar aún más la atención de las autoridades de los distintos niveles de gobierno a fin de que aceleren el ritmo en los procesos de concesiones e inversiones públicas de infraestructura.

El presente trabajo se estructura de la siguiente manera. Luego de la Introducción, en la sección II se revisa la literatura en que se aborda la relación entre infraestructura y crecimiento económico. Más adelante, en la sección III, se plantean las consideraciones metodológicas pertinentes. En la sección IV se analizan los resultados obtenidos de acuerdo con los modelos estimados. Finalmente, en la sección V se entregan las conclusiones y recomendaciones. 


\section{II}

\section{Revisión de la literatura}

\section{Marco teórico}

En el cuadro 1 se resumen los principales estudios en que se ha abordado la relación entre crecimiento económico e infraestructura. Existe consenso en considerar a la inversión en infraestructura pública como un componente importante del crecimiento económico. Este hecho fue inicialmente comprobado en la práctica por Aschauer (1989) y luego fue corroborado por autores como Easterly y Rebelo (1993); Canning (1999) y Calderón y Servén (2004b), así como por Vásquez y Bendezú (2008) para el caso peruano. Sin embargo, la discusión no parece centrarse en la dirección del efecto, sino en su magnitud. Así, por ejemplo, en la revisión de la literatura realizada por Straub (2008a), solo encontró un efecto negativo en el 6,5\% de los estudios, en todos los cuales se utilizó un indicador de infraestructura inadecuado (como se verá más adelante); por otra parte, en el 37,5\% de los estudios se obtuvieron resultados nulos, en comparación con el $55,8 \%$ en que se constataron coeficientes positivos.

El trabajo pionero de Aschauer (1989) es uno de los principales en que se ha encontrado evidencia empírica sobre el efecto positivo de la infraestructura en la producción. Este autor sostiene que la caída en la productividad del capital en los Estados Unidos de América durante las décadas de 1970 y 1980 obedeció a la reducción en la inversión pública en infraestructura. En particular, el autor afirma que las infraestructuras que afectan en mayor medida a la productividad son las de transportes (carreteras, puertos y aeropuertos), energía y saneamiento.

El primer paso para iniciar la discusión de la literatura a nivel teórico es plantear una función de producción en que se incluya explícitamente la variable de interés, el acervo (stock) de infraestructura pública, de manera similar a como lo hace Straub (2008a):

$$
Y=A(\theta, F) . F(K, L, I(N))
$$

donde $Y$ es el producto agregado, $A$ el término de productividad, $K$ el acervo de capital (sin contabilizar la infraestructura), $L$ el factor trabajo, e $I(N)$ una variable de insumos (inputs) intermedios en la que la infraestructura pública $(N)$ es la variable determinante. El nivel de infraestructura se halla separado de $K$, donde normalmente se encuentra incluido, tal como $I(N)$ refleja el efecto directo de $N$. Al incluir la infraestructura como un factor explicativo de $A$ (el efecto indirecto de $N$ ), se asume que la infraestructura tiene un efecto en la productividad total de factores. $\theta$ da cuenta de todos los otros factores que afectarían al término de productividad.

Teóricamente, es preferible modelar el efecto directo de la infraestructura a través de los servicios que esta provee $I(N)$, en lugar de incluirla directamente en la función de producción. En primer lugar, y tal como señalan Romp y De Haan (2007), incluir directamente la variable de infraestructura implicaría asumir que esta tiene atributos de bien público puro, y que provee servicios de manera proporcional a la cantidad de infraestructura, sin rivalidad ni exclusión en el consumo. En realidad, la infraestructura pública no produce nada en sí misma, simplemente provee servicios (como transporte y comunicaciones) que se incorporan dentro de las funciones de costos de las empresas (Hulten, Bennathan y Srinivasan, 2006).

En segundo lugar, Pritchett (1996) explica que las inversiones en infraestructura generalmente no se determinan mediante mecanismos de mercado, dado que suelen verse influenciadas por el marco regulatorio, que generalmente enfrenta problemas de información imperfecta (Laffont y Tirole, 1993); además, suelen ser susceptibles a la interferencia política (Guasch, Laffont y Straub, 2005), por tratarse en muchos casos de monopolios naturales. Ello se traduce en que las empresas no puedan tomar decisiones con respecto al costo de la cantidad de infraestructura que utilizan (Duggal, Saltzman y Klein, 1999; Hulten, Bennathan y Srinivasan, 2006).

Por otra parte, el efecto indirecto de la infraestructura implica asumir que su acumulación genera externalidades que incrementan la eficiencia general de la economía. Prud'homme (2005) argumenta que la infraestructura tiene un efecto similar al de la reducción de aranceles, pues permite aumentar el tamaño del mercado, lo que conlleva una mayor especialización, una competencia más intensa, economías de escala y el acrecentamiento del tamaño efectivo del mercado laboral. Duggal, Saltzman y Klein (1999) añaden que la infraestructura tiene también un importante efecto de red. Un ejemplo de ello se da en la calidad de la oferta eléctrica, que hace 
posible que se utilicen maquinarias más sofisticadas (Hulten, Bennathan y Srinivasan, 2006).

Asimismo, la infraestructura pública se diferencia del capital en general en otros aspectos adicionales. Por una parte, la magnitud de la infraestructura suele ser significativa y una fracción de ella no es capaz de proveer servicio alguno; por tanto, se requiere que una obra de infraestructura esté completa para que sea útil. Ello implicará, en la mayoría de los casos, que se requiera de grandes inversiones y largos períodos de espera hasta poder recibir servicios por parte de dicha infraestructura.

En algunas oportunidades, la relación de la infraestructura pública con el nivel y variación de la producción puede ser ambigua, ya que existen ciertas obras de infraestructura desarrolladas exclusivamente para mejorar el bienestar de cierto grupo poblacional, dándole prioridad al criterio de redistribución por sobre el de eficiencia económica. Además, según lo señalado por Barro y Sala-i-Martin (1990) y Glomm y Ravikumar (1994), la infraestructura puede estar sujeta a congestión, por lo que su repercusión en la economía dependerá del nivel de congestión en un momento dado; sin embargo, si el incremento en el acervo de la infraestructura ocurre con respecto a una infraestructura no congestionada, no tendrá grandes beneficios, pues no mejorará considerablemente la calidad del servicio. Ello podría implicar que en algunos casos sea preferible invertir en el mantenimiento de la infraestructura existente en lugar de construir una nueva (Hulten, 1996).

La productividad de las inversiones en infraestructura dependerá en gran medida de otros cuellos de botella en la economía, como la calidad institucional - especialmente los mecanismos contractuales-, el nivel de competencia (empresas estatales, concesiones, asociaciones público-privadas, y otras) y el mecanismo de aprobación de los proyectos.

Otro punto de discusión importante se refiere a la duración de los efectos de las infraestructuras nuevas o, dicho en otros términos, si esos efectos serán permanentes o transitorios. De acuerdo con Straub (2008b), lo primero implica suponer que la infraestructura genera suficientes externalidades para inducir retornos constantes a escala en términos agregados, por lo que se trataría del caso del crecimiento endógeno. Por otra parte, suponer que los efectos son transitorios implica que cualquier inversión en infraestructura tendrá rendimientos decrecientes, por lo que se aplicaría el caso neoclásico de crecimiento exógeno en el que la infraestructura afecta al producto, pero no a la tasa de crecimiento de largo plazo.

Según Mankiw, Romer y Weil (1992), la evidencia empírica sostiene que la variación en el producto se explica de manera adecuada manteniendo el supuesto de los retornos decrecientes a escala del capital. Por lo tanto, no debería descartarse la posibilidad de analizar las diferencias en el producto a partir de un modelo de Solow típico (Solow, 1956).

Finalmente, desde un punto de vista de políticas públicas es interesante discutir la posibilidad de la existencia de un punto óptimo para el acervo de infraestructura. Esto se podría hallar con facilidad igualando su costo marginal a sus beneficios sociales. Sin embargo, la determinación del costo de la infraestructura constituye un reto importante.

\section{Técnicas utilizadas}

Si bien la relación teórica entre la inversión en los distintos tipos de infraestructura pública y el crecimiento del producto interno bruto (PIB) ha sido explicada con bastante claridad en los numerosos estudios sobre el tema, el debate surge al tratar de plasmar esta relación teórica en un modelo econométrico. Esto se debe principalmente a tres puntos: i) ¿cómo lidiar con la endogeneidad existente entre la inversión en infraestructura y el crecimiento del producto?; ii) ¿qué medida de infraestructura es la que refleja su verdadero efecto en el producto agregado? y, posteriormente, ¿cómo separar el efecto que tiene cada tipo de infraestructura en el producto?, y, por último, iii) ¿qué controles adicionales se deben introducir a fin de no confundir el efecto de la infraestructura con el de otras variables vinculadas al entorno económico y político del país?

En el cuadro 1 se presentan los resultados de los principales estudios revisados en relación con el aporte de la infraestructura al crecimiento económico. En los primeros estudios se estima una regresión lineal simple, tomando como base un indicador monetario del gasto en infraestructura. Muchos autores atribuyen a este tipo de aproximación el hecho de que tanto Aschauer (1989) como Munnell (1990) hayan obtenido elasticidades tan grandes para la infraestructura. En estudios posteriores, como los de Devarajan, Swaroop y Zou (1996) y GarcíaMilà, McGuire y Porter (1996), se obtienen resultados menores o incluso negativos, utilizando un modelo de panel de datos con efectos fijos que capturan las diferencias no observadas entre los países. No obstante, Canning (1999), Calderón y Servén (2004b) y Straub, Vellutini y Warlters (2008) obtienen coeficientes mayores para la inversión en infraestructura al utilizar un indicador físico para su medición. En otros estudios, como los de Rivera y Toledo (2004) y Vásquez y Bendezú (2008), se encuentra una relación de cointegración entre las 
CUADRO 1

Resumen de principales estudios

\begin{tabular}{|c|c|c|c|c|c|}
\hline Estudio & Países evaluados & Muestra & Variable de infraestructura & Metodología & Elasticidad \\
\hline Aschauer (1989) & Estados Unidos de América & $1949-1985$ & $\begin{array}{l}\text { Gasto público en capital } \\
\text { no militar }\end{array}$ & $\mathrm{MCO}$ & 0,39 \\
\hline Munnell (1990) & Estados Unidos de América & $1947-1988$ & $\begin{array}{l}\text { Gasto público en } \\
\text { infraestructura }\end{array}$ & $\mathrm{MCO}$ & 0,34 \\
\hline Canning (1999) & 57 países en desarrollo & $1960-1990$ & Telecomunicaciones & Panel de efectos fijos & 0,139 \\
\hline $\begin{array}{l}\text { Easterly y Rebelo } \\
\text { (1993) }\end{array}$ & 28 países en desarrollo & $1970-1988$ & $\begin{array}{l}\text { Gasto en transporte y } \\
\text { telecomunicaciones }\end{array}$ & MCO & 0,16 \\
\hline $\begin{array}{l}\text { Esfahani y Ramírez } \\
\text { (2003) }\end{array}$ & 75 países & $1965-1995$ & $\begin{array}{l}\text { Telecomunicaciones y } \\
\text { energía }\end{array}$ & $\begin{array}{l}\text { MCO } \\
2 \text { etapas }\end{array}$ & $\begin{array}{l}0,091 \mathrm{y} \\
0,156\end{array}$ \\
\hline $\begin{array}{l}\text { Vásquez y Bendezú } \\
\text { (2008) }\end{array}$ & Perú & $1940-2003$ & Caminos & Cointegración & 0,218 \\
\hline $\begin{array}{l}\text { Rivera y Toledo } \\
(2004)\end{array}$ & Chile & $1975-2000$ & $\begin{array}{l}\text { Inversión sectorial en } \\
\text { infraestructura }\end{array}$ & Cointegración & 0,16 \\
\hline $\begin{array}{l}\text { Sánchez-Robles } \\
\text { (1998) }\end{array}$ & $\begin{array}{l}57 \text { países } \\
19 \text { países de América Latina }\end{array}$ & $1970-1985$ & Índice de infraestructura & $\mathrm{MCO}$ & $\begin{array}{l}0,009 \\
0,012\end{array}$ \\
\hline $\begin{array}{l}\text { Devarajan, Swaroop y } \\
\text { Zou (1996) }\end{array}$ & 43 países en desarrollo & $1970-1990$ & $\begin{array}{l}\text { Gasto en transporte y } \\
\text { telecomunicaciones }\end{array}$ & Panel de efectos fijos & $-0,025$ \\
\hline $\begin{array}{l}\text { Calderón y Servén } \\
\text { (2004b) }\end{array}$ & 101 países & $1960-2000$ & Índice de infraestructura & $\begin{array}{l}\text { Panel de efectos fijos } \\
\text { MGM }\end{array}$ & $\begin{array}{l}0,0195 \\
0,0207\end{array}$ \\
\hline $\begin{array}{l}\text { Duggal, Saltzman y } \\
\text { Klein (1999) }\end{array}$ & Estados Unidos de América & 1960-1989 & $\begin{array}{l}\text { Gasto público en caminos } \\
\text { y estructuras }\end{array}$ & $\begin{array}{l}\text { MCo, especificación } \\
\text { no lineal }\end{array}$ & 0,27 \\
\hline $\begin{array}{l}\text { García-Milà, McGuire } \\
\text { y Porter (1996) }\end{array}$ & $\begin{array}{l}\text { Estados Unidos de América } \\
\text { (48 estados) }\end{array}$ & $1971-1983$ & $\begin{array}{l}\text { Gasto público en agua y } \\
\text { desagüe y autopistas }\end{array}$ & Panel de efectos fijos & $-0,058$ y $-0,029$ \\
\hline
\end{tabular}

Fuente: elaboración propia.

MCO: mínimos cuadrados ordinarios.

MGM: método generalizado de momentos.

variables de infraestructura y el crecimiento del producto mediante el método de Johansen y, posteriormente, estos autores intentan hallar la relación de corto plazo en un modelo de corrección de errores (véase el cuadro 1).

\section{Endogeneidad del modelo}

Con respecto a la primera controversia, en la mayoría de los estudios se mencionan tres principales fuentes de endogeneidad: la presencia de efectos fijos no observados en modelos aplicados a muchos países o regiones, la existencia de doble causalidad entre el producto y la inversión en infraestructura, y la abundancia de problemas relacionados con variables omitidas por el modelo y con un error de medición en las variables de infraestructura.

Tanto en el trabajo de Aschauer (1989), en que se utiliza información regional de los Estados Unidos de América, como en el de Munnell (1990), donde se elabora un panel de distintos países, se encontraron 
valores muy altos para la elasticidad del producto con respecto a la infraestructura $(0,31$ en el primer caso, y 0,54 en el segundo). Gramlich (1994) señala que estos resultados no concuerdan con la realidad, dado que tales elasticidades implicarían un retorno marginal de $100 \%$, es decir, las obras de infraestructura cubrirían la totalidad de sus costos en un año.

En estudios posteriores, como los de Holtz-Eakin (1994) y García-Milà, McGuire y Porter (1996), se señala que estos elevados resultados se deben a la omisión de un efecto fijo que tome en cuenta efectos no observados entre los países o regiones analizados. En estos estudios se encuentran resultados considerablemente menores que en los estudios de primera generación. Straub (2008b) revisa 51 estudios en que se aplica un panel de datos —en 25 de los cuales se incluyen efectos fijosy constata que en aquellos en que se aplicaron efectos fijos suele encontrarse, en promedio, un menor efecto de la infraestructura en el producto.

La segunda posible manifestación de la endogeneidad en estos modelos es la presencia de doble causalidad entre las variables de inversión en infraestructura y el producto, lo que puede sesgar los resultados hacia arriba, aun en el caso que se incluya explícitamente un efecto fijo. Lo ideal es poder aplicar una prueba que indique la dirección de la causalidad de una manera no ambigua. Desafortunadamente, en la mayoría de los casos esa prueba no se puede realizar debido a la naturaleza de la información, por lo que se debe buscar una solución alternativa.

Una primera opción es la adoptada por Canning y Pedroni (2004), quienes optan por utilizar un panel de datos. Ellos encuentran que la relación de largo plazo y la corrección de corto plazo entre la infraestructura y el producto no es similar entre distintos países. Al hallar que ambas variables no son estacionarias, pero están cointegradas, pueden estimar un modelo de corrección de errores sin incluir restricciones a priori. Posteriormente, mediante la introducción de restricciones en el modelo, se puede determinar en qué dirección fluye la causalidad.

Rivera y Toledo (2004) y Vásquez y Bendezú (2008) siguen este camino e intentan hallar la existencia de una relación de cointegración entre las variables de infraestructura $(N)$ y el producto $(Y)$. En ese sentido, se deben realizar — en primer lugar- pruebas de raíz unitaria para descartar efectivamente la presencia de un componente tendencial o un quiebre estructural en las series. En ambos casos, se encontró que cada variable presentaba una raíz unitaria.

Otro posible enfoque es resolver un sistema de ecuaciones simultáneas en el que se incluya una ecuación que explique el PIB y otra que explique la infraestructura. En este caso, el problema consistiría en determinar la forma funcional de la segunda ecuación, dado que los componentes que generan la inversión en infraestructura pueden variar a través de los países.

Algunos autores han optado por solucionar la endogeneidad tomando las primeras diferencias de los componentes, de tal manera que se pueda analizar el efecto de retroalimentación del crecimiento del producto en la inversión en infraestructura, tal como lo hicieron González, Guasch y Serebrisky (2007) y Esfahani y Ramírez (2003). En el caso de un panel de datos, esta diferenciación también ayuda a eliminar el componente no observado correspondiente a cada uno de los $n$ países analizados. Sin embargo, este enfoque no permite comprobar la relación de largo plazo existente entre ambas variables de interés y deja de lado la posibilidad de que las series estén cointegradas.

Aschauer (1989) y Calderón y Servén (2004b) optan por utilizar instrumentos internos, como son los rezagos de las variables explicativas de interés. Esto es preferible a la opción anterior, porque estima explícitamente el proceso autorregresivo de las series. Con ello, se obtienen coeficientes robustos: en la mayoría de las investigaciones que siguieron esta metodología se obtuvieron coeficientes similares para la inversión en infraestructura con respecto a otros tipos de inversión en capital, y que además son consistentes con las tasas internas de retornos de muchos proyectos de transportes y telecomunicaciones del Banco Mundial, tal como lo señalan Bandyopadhyay y Devarajan (1993). Estos modelos son estimados paralelamente utilizando MCO y el método generalizado de momentos (MGM).

Una solución similar a la anterior es la empleada por Sánchez-Robles (1998) y Alesina y Perotti (1993), que consiste en utilizar variables de infraestructura medidas al inicio del período junto con variables de producto medidas al final del mismo período. Esta solución sigue un concepto similar a la instrumentalización interna, con la diferencia de que la elección de esa instrumentalización es preferible debido a que impone menos condiciones a priori con respecto a los instrumentos a utilizar.

Por otra parte, García-Milà, McGuire y Porter (1996) encuentran que en este tipo de investigaciones el hecho de emplear un panel cuadrado permite ahorrar el paso de instrumentalización interna. Esta observación es corroborada por Straub (2008b), quien muestra que en el caso de los estudios de paneles de datos la instrumentalización con rezagos propios no altera los resultados del modelo. 


\section{Elección de un indicador de infraestructura y separación de efectos}

En todos los estudios revisados se opta por incluir uno de dos tipos de indicadores de infraestructura: alguna medida monetaria de la inversión en infraestructura pública, o un índice físico de infraestructura relacionado con los servicios que provee.

Los indicadores del primer tipo suelen tomar como base la medición de la inversión en capital público. Sin embargo, dicha cuenta no necesariamente abarca de manera exclusiva lo invertido en infraestructura pública, sino que también puede comprender inversiones en edificios estatales y maquinaria estatal. Otra falla del indicador del primer tipo es que la participación del sector privado en la provisión de este tipo de infraestructura es cada vez más importante (el caso de las telecomunicaciones es evidente en el Perú), por lo que la medida de capital público sería insuficiente. Además, medir el monto invertido por las empresas privadas en infraestructura pública puede ser difícil de calcular, ya que las firmas suelen tratar de mantener sus estructuras de costos bajo la mayor reserva posible. Finalmente, a menudo el costo de la inversión no se relaciona con la cantidad de infraestructura que efectivamente se construye.

La elección de un indicador físico parece ser una alternativa preferible, pero no está exenta de problemas. La evidencia empírica es contundente al mostrar que los niveles de inversión en los distintos tipos de infraestructura pública se encuentran altamente correlacionados. Esto presenta una disyuntiva: incluir cada tipo de infraestructura pública por separado invalida el estimador MCO al reducir su eficiencia debido a la presencia de multicolinealidad, pero resumir dicha información en un solo indicador impide verificar qué tipo de inversión pública es la más productiva.

En la práctica, la mayoría de los autores — entre los que se encuentran Calderón y Servén (2004b) y SánchezRobles (1998) — han optado por intentar ambos enfoques, al estimar en paralelo una ecuación en que se incluye un índice agregado de infraestructura y otras en las que se incluye a la vez un tipo de infraestructura pública. Las diferencias de los coeficientes de los distintos tipos de infraestructura pública sirven para determinar cuál de ellos tiene la mayor productividad. En ambos casos, los índices se elaboran usando variables de tres sectores: transporte (longitud de red vial dentro del territorio total del país), generación eléctrica (capacidad de generación eléctrica en términos per cápita) y telecomunicaciones (número de líneas telefónicas en términos per cápita), por lo que un incremento en alguna de estas variables implicará un aumento en el valor del índice.

En lo que respecta al índice de infraestructura, la mayoría de los autores siguen a Alesina y Perotti (1993), quienes elaboraron un índice de inestabilidad sociopolítica basándose en el método de componentes principales. Con este método se procura resumir la información aportada por un conjunto de variables altamente correlacionadas en una sola variable (primer componente principal), que explica mejor la varianza de todas las series en conjunto. Para ello es importante que todas las variables tengan la misma dirección ${ }^{1}$, a fin de que la interpretación del principal componente pueda ser válida. En este caso, un incremento en el valor del componente principal implica un aumento de la inestabilidad política en términos generales (la manifestación específica de la inestabilidad política es irrelevante en este caso).

Limao y Venables (2001) utilizan un índice de infraestructura pública en un modelo con que se intenta explicar los costos de transporte. Para construir ese índice se emplearon las siguientes variables: kilómetros de carretera, kilómetros de carreteras asfaltadas, kilómetros de vías férreas (las tres divididas por el área del país) y número de líneas telefónicas por persona. Los autores sostienen que las cuatro variables listadas están altamente correlacionadas, y que no es posible identificar por separado el efecto de cada una en los costos de transporte. Ellos optan por utilizar el promedio lineal de las variables de infraestructura normalizadas, lo que implica asumir que los distintos tipos de infraestructura pública son sustitutos perfectos entre sí para una función de servicios de transporte. Los autores descartan aplicar el método de componentes principales, dado que la información emanada de dicha investigación presenta vacíos para muchos de los tipos de infraestructura analizados en distintos países.

Cabe resaltar que en el estudio elaborado por Calderón y Servén (2004b) se emplea información de América Latina, por lo que es el único estudio en que se ha construido un índice de infraestructura para el caso peruano, en este contexto. Asimismo, conviene señalar que en ninguno de los estudios examinados se realizó un análisis regional dentro de un mismo país, a excepción del de Vásquez y Bendezú (2008), precisamente para el caso peruano.

\footnotetext{
${ }^{1}$ La escala de medición y la magnitud no serían tan relevantes, puesto que las variables pueden ser normalizadas con facilidad.
} 
Antes de discutir qué variables adicionales se requieren para evitar que se sobreestime el efecto de la infraestructura a causa de la omisión de otras variables relevantes, es importante determinar cómo se pueden separar los efectos directos e indirectos de la infraestructura en el crecimiento. La literatura en que se intenta abordar frontalmente este punto es escasa. En la mayoría de los casos se suele utilizar una función de Cobb-Douglas, que no permite explicar los efectos indirectos en general. Los métodos de contabilidad del crecimiento tampoco posibilitan distinguir tales efectos, dado que - como se discutió anteriormente- no es fácil atribuirle un precio a la infraestructura de capital. Efectivamente, al ser la infraestructura un bien parcialmente público, su contribución al producto solo puede ser estimada de manera parcial.

De los estudios revisados, el único en que se modela el aporte de la infraestructura a través de su efecto indirecto es el de Duggal, Saltzman y Klein (1999). Estos autores utilizan un modelo no lineal e incluyen un índice de infraestructura como un factor adicional del residuo de Solow. Ellos hallan que el efecto de la infraestructura es positivo y de magnitud similar a la encontrada por Aschauer (1989), y que este efecto se retroalimenta positivamente con el progreso tecnológico.

\section{Elección de variables de control}

El último punto de la discusión metodológica se refiere al papel que cumplen las variables de control para la correcta especificación del modelo. Existen dos tipos de variables que permiten corregir posibles problemas en la especificación: variables ficticias (dummies), que recojan la presencia de quiebres estructurales, y variables relacionadas con el ciclo económico. Ello es necesario debido a que muchas veces el efecto de los parámetros varía a lo largo del tiempo, o estos siguen un ciclo determinado. Esta consideración es especialmente relevante para estudios regionales o estudios de corte transversal.

Con respecto al primer punto, Rivera y Toledo (2004) y Vásquez y Bendezú (2008) realizan pruebas para detectar la presencia de un quiebre estructural en el caso chileno y en el caso peruano, respectivamente. Esto se debe a los cambios en el tipo de gestión de las entidades encargadas de realizar la inversión en infraestructura pública en estos países, los que se produjeron al inicio de los años noventa. En ambos trabajos se incluye dicha variable en el modelo de corrección de errores estimado como resultado de un proceso de cointegración.

Vásquez y Bendezú (2008) también incluyen como controles adicionales el ciclo fiscal y el ciclo de exportaciones, los que fueron calculados mediante el filtro de Hodrick y Prescott para eliminar la tendencia de las series de gasto fiscal y exportaciones, respectivamente. Ello, con tal de explicar correctamente las fluctuaciones del PIB en el corto plazo.

Este procedimiento está en concordancia con Canning (1999), quien incluyó una serie de variables ficticias para controlar por la fase del ciclo económico en que se hallaba la economía. Sin embargo, no debe confundirse la variable empleada por Canning (1999) con la variable ficticia empleada por Rivera y Toledo (2004) y Vásquez y Bendezú (2008) para corregir quiebres estructurales. Con la variable de Canning (1999) se intenta reflejar únicamente la fase del ciclo económico, lo que guarda relación con las variables de los ciclos fiscales y exportadores empleadas por Vásquez y Bendezú (2008). Por su parte, las variables ficticias utilizadas por Rivera y Toledo (2004) y Vásquez y Bendezú (2008) se refieren a cambios en el tipo de administración de las obras de infraestructura pública. 


\section{III}

\section{Consideraciones metodológicas}

\section{Modelos y métodos de estimación}

El punto de partida se encuentra en la ecuación (1) mostrada en la sección anterior, a partir de la cual se desagrega el acervo de capital en tres componentes: capital físico distinto a infraestructura (C), capital humano $(\mathrm{H})$ e infraestructura de servicios públicos $\mathrm{I}(\mathrm{N})$. De esta manera, la nueva expresión es la siguiente:

$$
Y_{i t}=A_{i t} \cdot H_{i t}^{\beta} \cdot C_{i t}^{\alpha} \cdot I(N)_{i t}^{\gamma} \cdot L_{i t}^{1-\alpha-\beta-\gamma} \cdot U_{i t}
$$

donde los supraíndices $\alpha, \beta, \gamma$ y "1- $\alpha-\beta-\gamma$ " reflejan las participaciones de cada tipo de factor en la función de producción: capital físico, capital humano, infraestructura de servicios públicos y mano de obra respectivamente. Si la suma de estas participaciones fuese 1 (uno), la función de producción tendría retornos a escala constantes; si fuese menos de 1 (uno) tendría retornos a escala decrecientes y si sumase más de 1 (uno) tendría retornos crecientes.

Del mismo modo, los subíndices $i$ y $t$ corresponden al individuo $i$ (cada región peruana) en el período $t$. Por último, se agrega el término $U_{i t}$ correspondiente al error.

Al tomarse los logaritmos a ambos lados de la ecuación (2), se tiene la siguiente expresión (que se aproxima en términos per cápita):

$$
y_{i t}=a_{i}+b_{t}+\beta h_{i t}+\alpha c_{i t}+\gamma I(n)_{i t}+u_{i t}
$$

donde los dos primeros términos de esta ecuación corresponden a la productividad total de los factores $(A)$, tal como Canning (1999) señala:

$$
A_{i t}=a_{i}+b_{t}
$$

De esta manera, $A_{i t}$ está conformada por el término $a_{i}$, que es el factor constante y específico de cada región (heterogeneidad no observable), y $b_{t}$, que es el crecimiento de la productividad, común para todas las regiones en el período $t$.

Así, realizar una estimación del panel de datos con efectos fijos permitiría a cada región tener su propio nivel de productividad total de factores sin la necesidad de calcularlo. Más bien, intentar incluir la mejora productiva de manera explícita podría generar errores de medición al estimarse de la manera convencional a través del residuo de Solow (1956).

No obstante, es necesario tener $h_{i t}$, que es el indicador de capital humano; $c_{i t}$, que es el acervo de capital físico distinto a infraestructura, y $n_{i t}$, que es la infraestructura de servicios públicos. Ahora bien, como en el presente trabajo se desea estimar el efecto de tres tipos de infraestructura de servicios públicos en la producción per cápita, el término $n_{i t}$ (que parte de la función $I(n)$ asumida) puede desagregarse en los siguientes componentes:

$$
n_{i t}=e_{i t}+t_{i t}+r_{i t}
$$

donde $e_{i t}, t_{i t}, \mathrm{y} r_{i t}$ son los indicadores que reflejan la infraestructura eléctrica, de telecomunicaciones y de redes viales, respectivamente, de cada región en cada momento. Se opta por utilizar únicamente estas infraestructuras, debido a las restricciones de información existentes para las demás infraestructuras a nivel regional y a sus características. Por una parte, no se cuenta con información de acceso al agua y saneamiento en una serie larga a nivel regional. Por otra, la fuerte centralización de la actividad portuaria y aeroportuaria en la capital del país hace que la actividad en los puertos y aeropuertos de las demás regiones sea reducida y resulte poco significativa para explicar el producto a nivel regional. La infraestructura ferroviaria, a su vez, no tiene tanta relevancia en términos productivos con relación a los caminos y carreteras en las regiones del Perú. Finalmente, la reducida penetración de la infraestructura de banda ancha y el escaso acceso a gas natural (estas infraestructuras se vienen desarrollando hace menos de 10 años en el país y el gas natural se provee únicamente en la capital) inciden en que no sean aún tan relevantes para explicar las diferencias en los productos a nivel regional.

Cabe subrayar que en esta especificación se asume que la función $I(n)$ es lineal. Esto supone que no existen complementariedades entre los distintos tipos de infraestructura, lo que no es necesariamente cierto, porque algunas infraestructuras como las telecomunicaciones requieren de la presencia de energía eléctrica para operar. Sin embargo, se asume la relación lineal dado que se procura analizar la contribución de cada tipo de infraestructura al crecimiento productivo regional, y estimar 
otro tipo de función podría distorsionar los resultados ya que parte de la repercusión de cada infraestructura en el producto sería absorbida por otra infraestructura.

La ecuación (3) se transforma entonces en la expresión (6), que es la que se estima en este estudio:

$$
y_{i t}=A_{i t}+\beta h_{i t}+\alpha c_{i t}+\delta e_{i t}+\lambda t_{i t}+\sigma r_{i t}+u_{i t}
$$

Para recoger también posibles repercusiones diferenciadas de las distintas infraestructuras en el producto per cápita de las regiones, se construyen variables dicotómicas (dummies) para cada región y se añaden interacciones entre estas variables y las variables de infraestructura en el modelo.

Este modelo es interesante, puesto que mostraría el efecto transitorio de la infraestructura en la actividad económica. De acuerdo con Straub (2008b) y Mankiw, Romer y Weil (1992), este tipo de modelos refleja la teoría neoclásica de crecimiento exógeno, donde la infraestructura afecta al producto y no a la tasa de crecimiento de largo plazo.

En esta especificación se utiliza el estimador de mínimos cuadrados ordinarios (MCO) y el estimador de variables instrumentales (IV) a través del método generalizado de momentos (MGM), asumiendo un pool de datos (se estima a través del estimador IV-MGM). Estos estimadores no consideran la endogeneidad entre las variables y la heterogeneidad no observable existente entre regiones, pero es conveniente incluirlos para evaluar la robustez de los resultados econométricos obtenidos. Además, este modelo se estima mediante el estimador de mínimos cuadrados generalizados (MCG) para panel estático, que sí incorpora la heterogeneidad no observable. En cambio, no se estima a partir del estimador intragrupos (de efectos fijos), debido a que este elimina la heterogeneidad no observable del modelo. Es importante que se controle por la heterogeneidad no observable bajo esta especificación, dado que ella recoge las repercusiones de la productividad en el producto. Para lo cual se asume que la heterogeneidad no observable no se encuentra correlacionada con las variables explicativas.

Con el fin de desarrollar un análisis más completo, también se estiman modelos en diferencias para identificar el efecto de las distintas infraestructuras en el crecimiento económico regional ${ }^{2}$.

\footnotetext{
2 Debido a que las variables se encuentran en logaritmos, al estimar el modelo en diferencias la variable dependiente se transforma en el crecimiento del producto per cápita regional y los coeficientes estimados serían las elasticidades.
}

$$
\begin{gathered}
\Delta y_{i t}=\Delta A_{i t}+\zeta \Delta h_{i t}+\eta \Delta c_{i t}+ \\
\psi \Delta e_{i t}+\omega \Delta t_{i t}+\phi \Delta r_{i t}+\Delta u_{i t}
\end{gathered}
$$

Este modelo se estima mediante MCO, IV-MGM, MCG (efectos aleatorios) y estimador intragrupos (efectos fijos). Este último se justifica porque el modelo se estima en diferencias; por lo tanto, se estima un modelo en que no se considera el componente no observable específico de cada región, relacionado con la productividad. Además, al estimar el modelo en diferencias se eliminan las posibles raíces unitarias vinculadas a las variables utilizadas en la especificación.

Es importante mencionar que, al eliminar el componente relacionado con la productividad bajo esta especificación, se estaría perdiendo la principal fuente que permite explicar el crecimiento económico bajo los modelos neoclásicos de crecimiento exógeno. Así, mediante este modelo se estaría evaluando indirectamente si el crecimiento productivo de largo plazo de las regiones es endógeno ${ }^{3}$ y si la infraestructura tiene un efecto significativo.

Asimismo, se estiman modelos de panel dinámico tomando como variable explicativa al rezago de la variable dependiente. Esta estimación se realiza a través del MGM en diferencia y en sistema:

$$
\begin{gathered}
\Delta y_{i t}=\alpha \Delta y_{i t-1}+\Delta A_{i t}+\zeta \Delta h_{i t}+ \\
\eta \Delta c_{i t}+\psi \Delta e_{i t}+\omega \Delta t_{i t}+\phi \Delta r_{i t}+\Delta u_{i t}
\end{gathered}
$$

El MGM en diferencia aplicado a los modelos de panel dinámico permite controlar por una potencial endogeneidad en las variables explicativas — dado que instrumentaliza las variables a partir de los primeros y segundos rezagos de las variables utilizadas en la estimación-y tomar en cuenta la posible persistencia de la variable dependiente (Arellano y Bond, 1991; Arellano y Bover, 1995; Blundell y Bond, 1998). Esto resulta importante para evaluar un modelo de crecimiento endógeno.

Bajo este estimador se asumen distintos conjuntos de condiciones de momentos que involucran a los niveles y diferencias de las variables explicativas como instrumentos adicionales. Esto de acuerdo con las recomendaciones de Ashauer (1989) y de Calderón y Servén (2004b) de utilizar un proceso de instrumentalización

\footnotetext{
3 Para abordar esta hipótesis más adecuadamente se requiere un cambio en la especificación del modelo, a fin de eliminar la presencia de rendimientos decrecientes a escala de los factores de producción. No obstante, este objetivo escapa a los alcances de este artículo.
} 
interna para abordar la relación entre infraestructura y crecimiento económico. El estimador bajo el MGM es el siguiente:

$$
\delta_{M G M}=\left(\Delta X^{\prime} Z W_{N} Z \Delta X\right)^{-1} \Delta X^{\prime} Z W_{N} Z^{\prime} \Delta Y
$$

En esta expresión, $Z$ es la matriz de instrumentos, $X$ es la matriz que contiene a las variables explicativas, $Y$ es la variable dependiente del modelo y $W_{N}$ es la matriz de ponderadores definida positiva $\left[\frac{1}{N} \sum_{i=1}^{N}\left(Z_{i}^{\prime} \Delta \hat{v}_{i} \Delta \hat{v}_{i}^{\prime} Z_{i}\right)\right]^{-1}$. En esta última expresión, se presenta a los $\Delta \hat{v}_{i}$, que son los estimados consistentes de las primeras diferencias de los residuos obtenidos de un estimador consistente previo. Por tanto, el estimador $\delta_{M G M}$ es un estimador de dos etapas.

Según Blundell y Bond (1998), el MGM de primeras diferencias presenta sesgo e imprecisión en muestras finitas cuando las variables rezagadas de las series se encuentran débilmente correlacionadas con las subsiguientes primeras diferencias, de modo que los instrumentos para las ecuaciones en primeras diferencias son débiles. Por tal razón, estos autores consideran mayores restricciones en el modelo a partir de las cuales se obtiene un número más elevado de condiciones de momento. Explotar las condiciones adicionales de momento en algunos casos puede generar un incremento significativo en la eficiencia y reducción del sesgo de selección (Blundell y Bond, 1998). Así, conviene estimar también el modelo a través del uso del estimador del MGM en sistema, que incluye una mayor cantidad de instrumentos.

Para evaluar la validez de los instrumentos empleados a partir del uso del estimador del MGM (en diferencia y en sistema), se utiliza la prueba de Sargan, es decir, un contraste de especificación que se emplea para comprobar la validez de las restricciones que "sobreidentifican" a las variables instrumentales. La hipótesis nula de esta prueba es la ausencia de correlación entre los instrumentos y los residuos en el modelo en primeras diferencias.

Finalmente, también se debe considerar la evaluación de la prueba de correlación serial (Arellano y Bond, 1991). Bajo la hipótesis nula de ausencia de correlación serial entre los errores de la estimación, se debe encontrar una correlación negativa significativa en los residuos de la ecuación en primeras diferencias y la ausencia de correlación de segundo orden en estos residuos. Solo en el caso de que se cumplan ambas condiciones a la vez, la especificación de panel dinámico utilizada será válida.

\section{Información y variables}

En el estudio se toma en consideración la información de las 24 regiones del Perú durante el período 19802009. Las fuentes de información son oficiales: Instituto Nacional de Estadística e Informática (INEI), Organismo Supervisor de Inversión Privada en Telecomunicaciones (OSIPTEL), Ministerio de Energía y Minas (MINEM) y Ministerio de Transportes y Comunicaciones (MTC), aunque en algunos casos se incluyeron supuestos y estimaciones propias para completar las series de datos.

Según el modelo descrito, las variables de infraestructura necesarias para la estimación son el número de líneas telefónicas en servicio (fijas y móviles) como indicador de la infraestructura en telecomunicaciones, la potencia eléctrica instalada como indicador del sector electricidad y un indicador de las carreteras en estado asfaltado, pavimentado o ripiado sobre el total de carreteras existentes en cada región.

Cabe precisar que para que las estimaciones reflejen la verdadera repercusión de la infraestructura en el producto es necesario ajustarlas primero por posibles problemas de escala. Por ejemplo, la diferencia en tamaños de población de las regiones justifica una cantidad más elevada de líneas telefónicas en las regiones más pobladas, sin que ello conlleve una mayor capacidad relativa de proveer un servicio y, por ende, no determina una tasa más alta de crecimiento o tamaño del producto. Del mismo modo, si se diera una mayor longitud de la red vial en las regiones más extensas, ello no tendría necesariamente como consecuencia una productividad más elevada de este indicador en comparación con las otras regiones.

Debido a esto, se procedió a dividir la serie de valor bruto de la producción — que es empleada como variable sustitutiva (proxy) del PIB- entre la población de la región. Esta es la variable dependiente usada regularmente en este tipo de estudios, y ha sido incluida en los logaritmos. De manera similar, los indicadores de telecomunicaciones y de electricidad se expresaron en términos per cápita para cada región.

El indicador de infraestructura de transporte se construyó como el cociente entre el logaritmo de la longitud de la red vial asfaltada, pavimentada o ripiada sobre la superficie de la región para corregir por escala, y el logaritmo de la longitud de la red vial total también corregido por escala. Este es un indicador relativo de capacidad mediante el cual no solo se toman en cuenta las diferencias de superficie, sino también la diferencia en la calidad de la red vial. Conviene resaltar que este indicador no es el empleado en los otros estudios, donde más bien se utiliza el logaritmo de carreteras asfaltadas, 
pavimentadas o ripiadas sobre la superficie del país bajo análisis. Se opta por este indicador debido a la poca variación que ha presentado el nivel de carreteras (total y asfaltado) sobre la superficie de las regiones peruanas en la última década. Una de las principales limitaciones de esta elección es que no permite que se compare el coeficiente vinculado a este tipo de infraestructura con los de las infraestructuras de electricidad y telecomunicaciones.

Las demás variables utilizadas, con fines de control, son la población económicamente activa (PEA) ajustada por capital humano y el acervo de capital distinto de la infraestructura. La primera variable puede estimarse por medio de la PEA con nivel secundario, superior o ambos, o con los años de escolaridad promedio, que suele ser la más utilizada en los estudios internacionales. En el presente trabajo se empleó la PEA con nivel secundario o superior debido a la falta de estadísticas oficiales respecto de los años de escolaridad promedio de la PEA por región. Además, se maneja como indicador alternativo a la PEA en niveles, en caso de que la PEA con nivel secundario no sea significativa bajo algunas especificaciones. Ambos indicadores se estandarizan dividiéndolos entre la población de la región.

Con respecto al acervo de capital distinto de la infraestructura, se utilizó la variable sustitutiva (proxy) elaborada por Vásquez y Bendezú (2008) para los años 1973 y 1993, y se siguió su metodología en la elaboración de esta variable para el año 2007 sobre la base del IV Censo Nacional Económico del INEI. La metodología propuesta por estos autores consiste en recopilar el valor de los activos fijos a nivel regional de empresas comerciales e industriales (costo inicial, revalorización y depreciación acumulada). La construcción de la serie regional para los años intercensales se realizó según el patrón de la inversión en cada región.

No obstante, como en esta serie se incluye la inversión privada en electricidad, telefonía y carreteras (lo que podría distorsionar los resultados del modelo estimado) se procedió a limpiarla de estos componentes. Para ello, se estimó un modelo mediante MCO cuya variable dependiente es el acervo de capital estimado y cuyas variables explicativas son las tres variables de infraestructura elegidas. El residuo de esa estimación se toma como variable sustitutiva del capital distinto de la infraestructura, luego de dividirlo entre la población y aplicarle logaritmos.

Ahora bien, para que los coeficientes estimados sean insesgados y eficientes es recomendable que las variables que lo conforman sean estacionarias y no presenten quiebre estructural. A fin de detectar estos problemas se realizaron las pruebas de Levin, Lin y Chu (2002) y de Im, Pesaran y Shin (2003), ambas desarrolladas en Cameron y Trivedi
(2009). Estas pruebas presentan como hipótesis nula que todos los paneles tienen raíz unitaria. En la prueba de Levin, Lin y Chu se asume un mismo parámetro autorregresivo para todos los paneles, es decir, una raíz unitaria común para todos los individuos (regiones), por lo que rechazar la hipótesis nula podría significar que algunas regiones efectivamente tengan series que presentan raíz unitaria y que otras regiones tengan la misma serie, pero con un comportamiento estacionario. La especificación de la prueba para la existencia de intercepto individual para cada observación, pero sin tendencia, requiere que el número de períodos crezca más rápido que el de las regiones, por lo que la razón de regiones a períodos tiende a 0 (cero) empleando pruebas de Dickey-Fuller aumentadas para cada región. De modo que al rechazar la hipótesis nula (con un nivel de significancia de 5\%) se acepta que en ningún caso existe raíz unitaria común para todos los individuos.

Asimismo, como recomienda Canning (1999), para comprobar la "estacionariedad" media de las series se utilizó una prueba más potente que la de Dickey-Fuller aumentada, mediante el procedimiento secuencial de Dolado, Jenkinson, y Sosvilla-Rivero (1990) ${ }^{4}$ aplicado a cada serie para cada individuo (región). En este test de Im, Pesaran y Shin (2003) también se utiliza la prueba de Dickey-Fuller, pero se promedian los estadísticos-t encontrados, de modo que el estadístico-t resultante represente al promedio de tendencias estocásticas independientes ${ }^{5}$ de una distribución con valor esperado y varianza conocida para una serie no estacionaria. Cabe subrayar que aunque esta prueba también está diseñada para paneles donde haya más períodos de tiempo que individuos, se constata que tiene alta potencia incluso en paneles cortos (Canning, 1999).

Los resultados de la aplicación de los tests no son contundentes para rechazar la hipótesis nula de no estacionariedad respecto de todas las variables. Esto es lógico en un contexto donde algunas infraestructuras, como la telefonía móvil, han tenido un avance significativo durante la última década en las regiones peruanas. Esta sería una de las principales limitaciones de las estimaciones. Sin embargo, dado que en los modelos en niveles no se incluye la variable dependiente rezagada, ello no sería una restricción relevante. Por otra parte, los modelos en diferencias eliminan la raíz unitaria, solucionando el problema de manera directa.

\footnotetext{
4 Tomado de Canning (1999).

${ }^{5}$ En otras palabras, permite la posibilidad de que los paneles no tengan raíces unitarias comunes.
} 


\section{Hechos estilizados de la economía peruana}

El crecimiento económico sostenido que ha experimentado la economía peruana durante la última década ha sido el más alto de la región y uno de los más elevados a nivel mundial. Este crecimiento se debe principalmente a las reformas de mercado desarrolladas en la década de 1990 y ha permitido una reducción significativa de la pobreza en el Perú.

Sin embargo, uno de los principales problemas que persiste en la economía peruana es la centralización de la producción y del desarrollo económico en Lima y en las principales regiones de la costa. Esto debido a que los esfuerzos por descentralizar la actividad productiva y fomentar el desarrollo de las regiones de la sierra y la selva han sido insuficientes. En 2009, Lima representó el $47 \%$ del valor bruto de producción del país, seguido por Arequipa, La Libertad y Piura con el 5,2\%, 4,4\% y $3,7 \%$ respectivamente. Por consiguiente, uno de los principales retos que presenta la economía peruana para la próxima década es que el desarrollo productivo alcance a las regiones más alejadas de la capital.
Por otra parte, existen algunas regiones que han presentado crecimientos económicos sostenidos debido al desarrollo de actividades productivas como la agroindustria (por ejemplo, Ica y Lambayeque, que presentan muy buenas condiciones climáticas y rendimientos agrícolas superiores a la mayoría de los países a nivel mundial) y la minería y los hidrocarburos (destaca Cusco con el Proyecto Camisea de gas natural). Así, el crecimiento económico experimentado por las regiones del Perú ha sido diferenciado, lo que se traduce en brechas importantes en los PIB per cápita actuales de las regiones (véase el gráfico 1).

Este desarrollo económico diferenciado se correlaciona estrechamente con la tenencia relativa de infraestructuras de uso público de cada región. En efecto, diversas regiones presentan una amplia brecha en el acceso a los distintos tipos de infraestructura, lo que constituye una de las principales trabas para el crecimiento económico de las localidades del interior del país (véase el gráfico 2).

La relación entre los distintos tipos de infraestructura y el producto per cápita de las regiones peruanas muestra una correlación positiva. Esta relación es

GRÁFICO 1

Perú: crecimiento acumulado del PIB, 2001-2009

(Variación porcentual del PIB real, a precios de 1994)

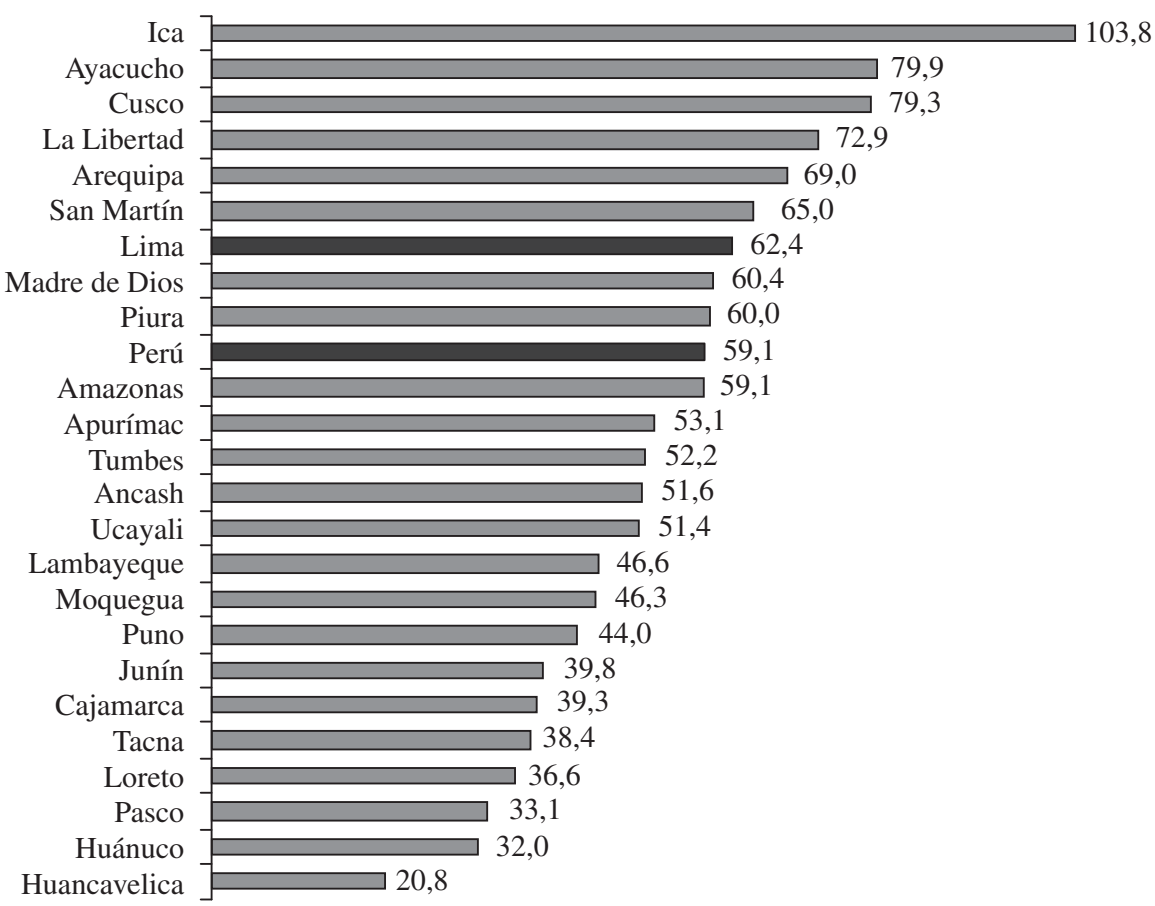

Fuente: elaboración propia sobre la base de datos del Instituto Nacional de Estadística e Informática (INEI).

PIB: producto interno bruto. 
GRÁFICO 2

Perú: PIB per cápita y acervo de infraestructura, 2009

Energía eléctrica

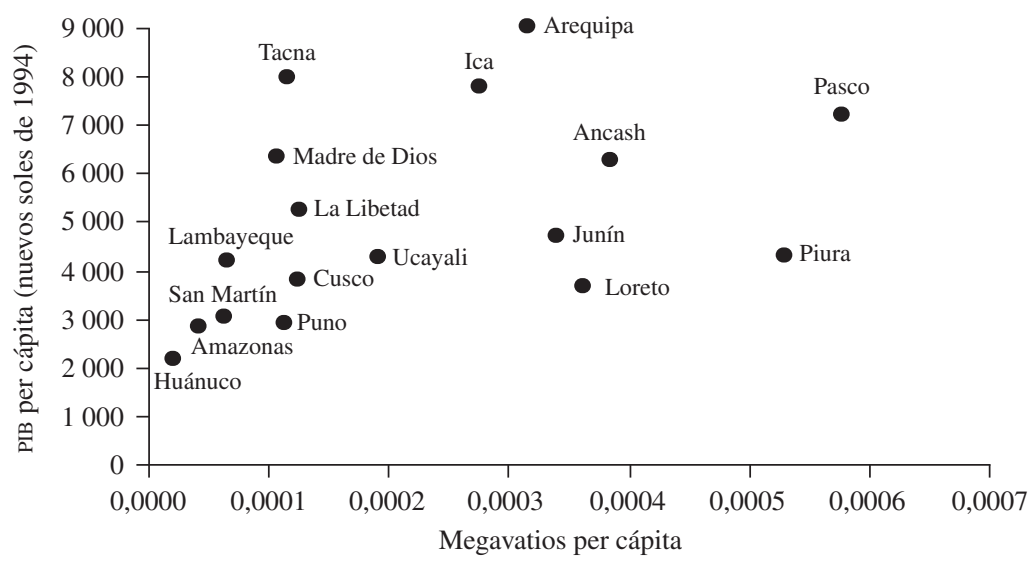

Carreteras

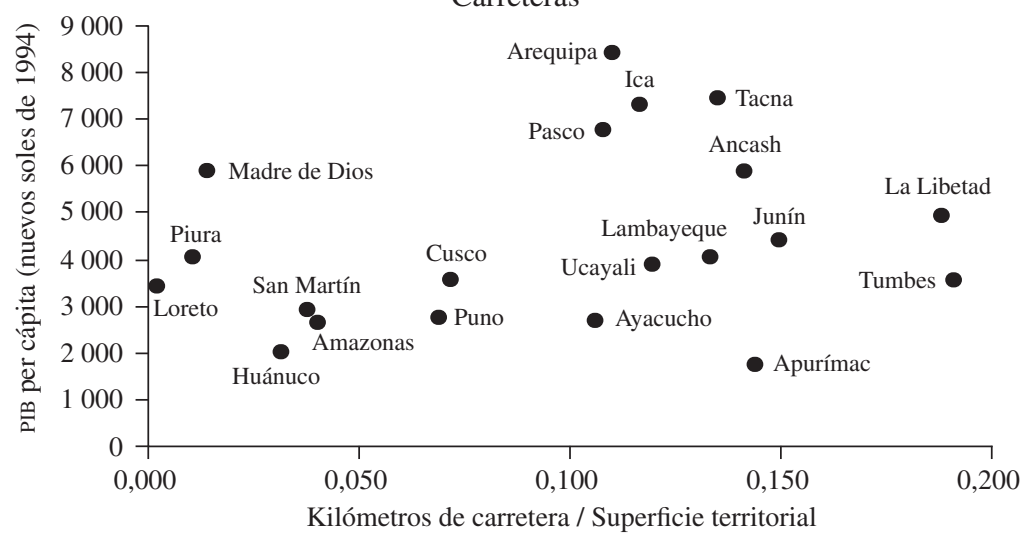

Telecomunicaciones

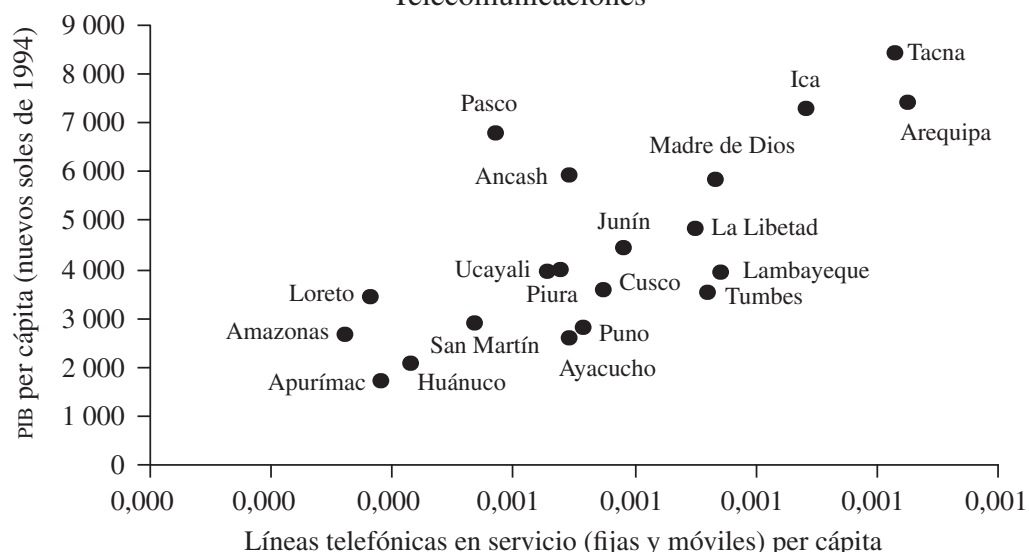

Fuente: elaboración propia sobre la base de datos del Instituto Nacional de Estadística e Informática (INEI), el Organismo Supervisor de Inversión Privada en Telecomunicaciones (OSIPTEL), el Ministerio de Energía y Minas (MINEM) y el Ministerio de Transportes y Comunicaciones (MTC). Se excluye a Lima para no distorsionar la relación existente.

PIB: producto interno bruto. 
más pronunciada en el caso de las infraestructuras de telecomunicaciones y energía eléctrica. De este modo, vale la pena analizar esta correlación mediante técnicas econométricas para encontrar qué tipo de infraestructura es la que tiene la mayor incidencia en la producción regional y el crecimiento económico.

\section{IV}

\section{Análisis de resultados}

En los modelos estimados se han considerado simultáneamente los indicadores para los sectores de energía, transportes y telecomunicaciones, tal como lo hicieron Canning (1999); Calderón y Servén (2004b); y Vásquez y Bendezú (2008). No obstante, en el presente trabajo no se ha controlado por la etapa del ciclo económico en que se encuentra la economía regional, a diferencia del primer estudio citado en que se utilizan controles para corregir dicho efecto, y del segundo estudio, donde se emplea una media móvil de cinco años. Se probó con la inclusión de variables dicotómicas temporales, pero estas resultaron no significativas.

\section{Modelos en niveles}

El primer grupo de modelos tiene al producto per cápita como variable dependiente y se estima en niveles, lo que se encuadra en la teoría neoclásica de crecimiento exógeno y evalúa los efectos transitorios de la infraestructura en el producto. Con estos modelos se verifica la presencia de un efecto positivo y significativo de todas las infraestructuras en el producto per cápita regional. Asimismo, esta relación prevalece a través de los diversos estimadores utilizados, lo que indica que los resultados son robustos (no existe un cambio en signos y las magnitudes de los coeficientes son similares entre especificaciones).

Para estos modelos, la PEA educada no resultó significativa, pero sí la PEA total cuyo coeficiente es el más alto. Esto muestra la importancia de este factor de producción dentro de la producción regional, en contraste con los demás factores de producción (capital físico, capital humano e infraestructuras) (véase al cuadro 2).

Cabe destacar que el acervo de electricidad per cápita es el que presenta el mayor efecto en el producto per cápita regional. Este resultado es lógico, debido a que la electricidad se relaciona directamente con la actividad productiva, mientras que las telecomunicaciones están mucho más ligadas a la productividad de los trabajadores. Por otra parte, el auge de las telecomunicaciones en el
Perú es un fenómeno registrado en la última década, por lo que al evaluarse un horizonte mayor se estaría reduciendo su repercusión.

Asimismo, es importante indicar que tanto el acervo de electricidad como el de telecomunicaciones presentan efectos mayores en el producto regional que el capital distinto de la infraestructura. Esto indicaría que las tenencias diferenciadas de acervos en infraestructura per cápita de las distintas regiones peruanas explican marcadamente las diferencias en los productos regionales per cápita. De esta manera, si las autoridades de política realizan esfuerzos por disminuir las brechas existentes en infraestructura entre las regiones se podría avanzar en la reducción de disparidades productivas.

Si bien el coeficiente relacionado con el indicador de acervo de carreteras no es comparable con los coeficientes de las demás infraestructuras, esta infraestructura explica una buena parte de las diferencias en la producción regional. Uno de los principales problemas a nivel regional es la falta de caminos y carreteras que permitan acercar los mercados a estas localidades. Entonces, resulta relevante priorizar la inversión en esta infraestructura.

Puede observarse cómo cada tipo de infraestructura explica de manera diferenciada el producto per cápita de cada región. Esto puede deberse a las diferencias en la calidad de la infraestructura regional, al capital humano en cada región (que no se controla en el modelo, pues la PEA con capital humano no resultó ser significativa) y a otras diferencias socioeconómicas o políticas que pudieran imponer trabas al correcto desempeño de las infraestructuras en algunas regiones. Estas diferencias son las que explican cómo algunas regiones pueden presentar efectos mayores o menores que el promedio de las distintas infraestructuras en el producto de sus respectivas regiones.

\section{Modelos en diferencias}

El segundo grupo de modelos tiene a la diferencia del producto per cápita como variable dependiente en 
CUADRO 2

\section{Modelo en niveles del logaritmo del PIB per cápita}

\begin{tabular}{|c|c|c|c|c|c|c|c|}
\hline Variable & $\begin{array}{l}\text { Pool de datos } \\
\text { (Estimador } \\
\text { MCO) }\end{array}$ & $\begin{array}{l}\text { Pool de datos } \\
\text { (Estimador } \\
\text { IV-MGM) }\end{array}$ & $\begin{array}{l}\text { Panel estático } \\
\text { (Estimador } \\
\text { MCG) }\end{array}$ & Variable & $\begin{array}{l}\text { Pool de datos } \\
\text { (Estimador- } \\
\text { MCO) }\end{array}$ & $\begin{array}{l}\text { Pool de datos } \\
\text { (Estimador } \\
\text { IV-MGM) }\end{array}$ & $\begin{array}{c}\text { Panel estático } \\
\text { (Estimador } \\
\text { MCG) }\end{array}$ \\
\hline Constante & $\begin{array}{l}10,9779 * * * \\
(0,1243)\end{array}$ & $\begin{array}{l}10,8915^{* * *} \\
(0,1470)\end{array}$ & $\begin{array}{l}10,9084 * * * \\
(0,3199)\end{array}$ & $\begin{array}{l}\text { Telecomunicaciones } \\
\text { (continuación) }\end{array}$ & & & \\
\hline Log [capital regional] & $\begin{array}{l}0,0607 * * * \\
(0,0123)\end{array}$ & $\begin{array}{l}0,0591 * * * \\
(0,0117)\end{array}$ & $\begin{array}{c}0,0543 \\
(0,0379)\end{array}$ & $\begin{array}{l}\text { Log [indicador telecom.] } \\
\text { *Madre de Dios }\end{array}$ & $\begin{array}{l}-0,0900 * * * \\
(0,0110)\end{array}$ & $\begin{array}{l}-0,0956^{* * *} \\
(0,0119)\end{array}$ & $\begin{array}{l}-0,0744 * * * \\
(0,0230)\end{array}$ \\
\hline $\log [\mathrm{PEA}]$ & $\begin{array}{l}1,3737 * * * \\
(0,1281)\end{array}$ & $\begin{array}{l}1,2960 * * * \\
(0,1464)\end{array}$ & $\begin{array}{l}1,4260 * * * \\
(0,3023)\end{array}$ & $\begin{array}{l}\text { Log [indicador telecom.] } \\
\text { *Pasco }\end{array}$ & $\begin{array}{l}-0,1397 * * * \\
(0,0078)\end{array}$ & $\begin{array}{l}-0,1405^{* * *} \\
(0,0076)\end{array}$ & $\begin{array}{l}-0,1358 * * * \\
(0,0115)\end{array}$ \\
\hline Electricidad & & & & $\begin{array}{l}\text { Log [indicador telecom.] } \\
\text { *Piura }\end{array}$ & $\begin{array}{l}0,0590 * * * \\
(0,0143)\end{array}$ & $\begin{array}{l}0,0591 * * * \\
(0,0141)\end{array}$ & $\begin{array}{l}0,0589 * * * \\
(0,0144)\end{array}$ \\
\hline Log [indicador electricidad] & $\begin{array}{l}0,1268 * * * \\
(0,0091)\end{array}$ & $\begin{array}{l}0,1265 * * * \\
(0,0089)\end{array}$ & $\begin{array}{l}0,1139 * * * \\
(0,0206)\end{array}$ & $\begin{array}{l}\text { Log [indicador telecom.] } \\
\text { *Puno }\end{array}$ & $\begin{array}{l}0,0470 * * * \\
(0,0080)\end{array}$ & $\begin{array}{l}0,0460^{* * * *} \\
(0,0077)\end{array}$ & $\begin{array}{l}0,0569^{* * *} \\
(0,0129)\end{array}$ \\
\hline $\begin{array}{l}\text { Log [indicador electricidad] } \\
\text { *Amazonas }\end{array}$ & & & $\begin{array}{l}0,0705 * * * \\
(0,0202)\end{array}$ & $\begin{array}{l}\text { Log [indicador telecom.] } \\
\text { *San Martín }\end{array}$ & $\begin{array}{l}-0,0646 * * * \\
(0,0174)\end{array}$ & $\begin{array}{l}-0,0655^{* * *} \\
(0,0168)\end{array}$ & $\begin{array}{l}-0,0581 * * * \\
(0,0123)\end{array}$ \\
\hline $\begin{array}{l}\text { Log [indicador electricidad] } \\
\text { *Apurímac }\end{array}$ & $\begin{array}{l}0,0101 * * * \\
(0,0038)\end{array}$ & $\begin{array}{l}0,0112 * * * \\
(0,0038)\end{array}$ & $\begin{array}{l}0,0132 * * * \\
(0,0051)\end{array}$ & $\begin{array}{l}\text { Log [indicador telecom.] } \\
\text { *Tacna }\end{array}$ & $\begin{array}{l}-0,2243 * * * \\
(0,0106)\end{array}$ & $\begin{array}{l}-0,1801 * * * \\
(0,0209)\end{array}$ & $\begin{array}{l}-0,2245^{* * *} \\
(0,0219)\end{array}$ \\
\hline $\begin{array}{l}\text { Log [indicador electricidad] } \\
\text { *Cajamarca }\end{array}$ & $\begin{array}{l}0,0478 * * * \\
(0,0136)\end{array}$ & $\begin{array}{l}0,0475^{* * *} \\
(0,0131)\end{array}$ & $\begin{array}{l}0,0541 * * * \\
(0,0112)\end{array}$ & Transportes & & & \\
\hline $\begin{array}{l}\text { Log [indicador } \\
\text { electricidad]*Cusco }\end{array}$ & $\begin{array}{l}0,0223 * * * \\
(0,0030)\end{array}$ & $\begin{array}{l}0,0220 * * * \\
(0,0030)\end{array}$ & $\begin{array}{l}0,0242^{* * * *} \\
(0,0029)\end{array}$ & Log [indicador transportes] & $\begin{array}{l}0,0395 * * * \\
(0,0055)\end{array}$ & $\begin{array}{l}0,0385 * * * \\
(0,0054)\end{array}$ & $\begin{array}{l}0,0331 * * \\
(0,0156)\end{array}$ \\
\hline $\begin{array}{l}\text { Log [indicador electricidad] } \\
\text { *Huánuco }\end{array}$ & $\begin{array}{l}0,0163 * * * \\
(0,0036)\end{array}$ & $\begin{array}{l}0,0171 * * * \\
(0,0034)\end{array}$ & $\begin{array}{l}0,0201 * * * \\
(0,0068)\end{array}$ & $\begin{array}{l}\text { Log [indicador transportes] } \\
\text { *Amazonas }\end{array}$ & & & $\begin{array}{l}-0,2428 * * * \\
(0,063)\end{array}$ \\
\hline $\begin{array}{l}\text { Log [indicador electricidad] } \\
\text { *Ica }\end{array}$ & $\begin{array}{l}-0,1226 * * * \\
(0,0452)\end{array}$ & $\begin{array}{l}-0,1160 * * * \\
(0,0442)\end{array}$ & $\begin{array}{l}-0,1184 * * * \\
(0,0398)\end{array}$ & $\begin{array}{l}\text { Log [indicador transportes] } \\
\text { *Cajamarca }\end{array}$ & $\begin{array}{l}-0,4364 * * * \\
(0,1493)\end{array}$ & $\begin{array}{l}-0,4309 * * * \\
(0,1444)\end{array}$ & $\begin{array}{l}-0,4908^{* * *} \\
(0,1423)\end{array}$ \\
\hline $\begin{array}{l}\text { Log [indicador electricidad] } \\
\text { *Moquegua }\end{array}$ & $\begin{array}{l}-0,0935^{* * *} \\
(0,0068)\end{array}$ & $\begin{array}{l}-0,0964 * * * \\
(0,0069)\end{array}$ & $\begin{array}{l}-0,0972 * * * \\
(0,0152)\end{array}$ & $\begin{array}{l}\text { Log [indicador transportes] } \\
\text { *Ica }\end{array}$ & $\begin{array}{l}0,6401 * * \\
(0,2959)\end{array}$ & $\begin{array}{l}0,5926 * * \\
(0,2895)\end{array}$ & $\begin{array}{l}0,6207 * * * \\
(0,2616)\end{array}$ \\
\hline $\begin{array}{l}\text { Log [indicador electricidad] } \\
\text { *San Martín }\end{array}$ & $\begin{array}{l}0,0389 * * * \\
(0,0067)\end{array}$ & $\begin{array}{l}0,0381 * * * \\
(0,0065)\end{array}$ & $\begin{array}{l}0,0421 * * * \\
(0,0078)\end{array}$ & $\begin{array}{l}\text { Log [indicador transportes] } \\
\text { *Lima }\end{array}$ & $\begin{array}{l}-1,3135^{* * *} \\
(0,1973)\end{array}$ & $\begin{array}{l}-1,3913 * * * \\
(0,2112)\end{array}$ & $\begin{array}{l}-1,3060^{* * *} \\
(0,2890)\end{array}$ \\
\hline Telecomunicaciones & & & & $\begin{array}{l}\text { Log [indicador transportes] } \\
\text { *Piura }\end{array}$ & $\begin{array}{l}-0,3007 * * * \\
(0,0613)\end{array}$ & $\begin{array}{l}-0,3002 * * * \\
(0,0603)\end{array}$ & $\begin{array}{l}-0,2927 * * * \\
(0,0212)\end{array}$ \\
\hline Log [indicador telecom.] & $\begin{array}{l}0,0757 * * * \\
(0,0077)\end{array}$ & $\begin{array}{l}0,0786 * * * \\
(0,0078)\end{array}$ & $\begin{array}{l}0,0680 * * * \\
(0,0178)\end{array}$ & $\begin{array}{l}\text { Log [indicador transportes] } \\
\text { *Tacna }\end{array}$ & & $\begin{array}{l}-0,0838^{* * *} \\
(0,0344)\end{array}$ & \\
\hline $\begin{array}{l}\text { Log [indicador } \\
\text { telecom.]*Arequipa }\end{array}$ & $\begin{array}{l}-0,0951 * * * \\
(0,0107)\end{array}$ & $\begin{array}{l}-0,0986 * * * \\
(0,0112)\end{array}$ & $\begin{array}{l}-0,0938 * * * \\
(0,0142)\end{array}$ & $\begin{array}{l}\text { Número de observaciones } \\
\mathrm{R}^{2}\end{array}$ & $\begin{array}{c}480 \\
0,9411\end{array}$ & $\begin{array}{c}480 \\
0,9414\end{array}$ & 480 \\
\hline $\begin{array}{l}\text { Log [indicador telecom.] } \\
\text { *Huánuco }\end{array}$ & $\begin{array}{l}-0,0332 * * * \\
(0,0100)\end{array}$ & $\begin{array}{l}-0,0345^{* * *} \\
(0,0097)\end{array}$ & $\begin{array}{l}-0,0326 * * * \\
(0,0108)\end{array}$ & $\mathrm{R}^{2}$ intragrupos & & & 0,6133 \\
\hline $\begin{array}{l}\text { Log [indicador telecom.] } \\
\text { *Loreto }\end{array}$ & $\begin{array}{l}-0,0954 * * * \\
(0,0104)\end{array}$ & $\begin{array}{l}-0,0934 * * * \\
(0,0103)\end{array}$ & $\begin{array}{l}-0,0827 * * * * \\
(0,0259)\end{array}$ & $\begin{array}{l}\mathrm{R}^{2} \text { entre grupos } \\
\mathrm{R}^{2} \text { promedio }\end{array}$ & & & $\begin{array}{l}0,9922 \\
0,9431\end{array}$ \\
\hline
\end{tabular}

Fuente: elaboración propia sobre la base de estadísticas oficiales del Instituto Nacional de Estadística e Informática (INEI), el Organismo Supervisor de Inversión Privada en Telecomunicaciones (OSIPTEL), el Ministerio de Energía y Minas (MINEM) y el Ministerio de Transportes y Comunicaciones (MTC) e información de Vásquez y Bendezú, Ensayos sobre el rol de la infraestructura vial en el crecimiento económico del Perú, Lima, Consorcio de Investigación Económica y Social (CIES)/Banco Central de Reserva del Perú (BCRP), 2008.

PIB: producto interno bruto. PEA: población económicamente activa. Log: logaritmo. MCO: mínimos cuadrados ordinarios. MCG: mínimos cuadrados generalizados.

Estimador de variables instrumentales (IV) a través del método generalizado de momentos (MGM).

Errores estándar entre paréntesis (robustos según White).

$* \mathrm{p}<0,10, * * \mathrm{p}<0,05, * * * \mathrm{p}<0,01$ 
función de las diferencias de las variables explicativas del modelo. Una de las principales ventajas que permite esta especificación es la eliminación de las raíces unitarias encontradas en las series. En contraposición, una de las principales desventajas es la pérdida en el ajuste de los modelos a lo largo del tiempo ( $\mathrm{R}^{2}$ intragrupos), aunque prevalece un ajuste moderado entre regiones $\left(\mathrm{R}^{2}\right.$ entre grupos), considerando que es un modelo de panel de datos estimado en diferencias.

Este resultado concuerda con los hallazgos encontrados por Mankiw, Romer y Weil (1992), quienes sostienen que la evidencia empírica respalda a los modelos neoclásicos de crecimiento exógeno. Esto explica el buen ajuste de los modelos en niveles en contraste con el ajuste de los modelos en diferencias. La pérdida en ajuste puede atribuirse a la ausencia de controles regionales (resultaron no significativas las variables dicotómicas vinculadas a cada región) y a la eliminación de la heterogeneidad no observable del modelo (eliminada al tomar diferencias al modelo, por ser invariante respecto del tiempo). Al estimar el modelo en diferencias, la última se vincula directamente a la productividad regional según la especificación del modelo, que recogería las principales diferencias en el crecimiento económico regional. Esto indicaría también que la infraestructura, el capital humano y el capital físico distinto de la infraestructura fallan parcialmente en explicar las diferencias en el crecimiento regional de largo plazo.

Además, esta evidencia sugiere la existencia de otros factores que son más importantes para explicar estas diferencias. Uno de ellos puede ser la casi inexistente descentralización de la producción. Asimismo, la presencia en algunas regiones de proyectos de hidrocarburos, minería o agroexportación también explicaría estas diferencias. Las actividades productivas relacionadas con estos sectores se han impulsado durante los últimos años debido a los elevados precios internacionales de algunos productos de agroexportación, hidrocarburos y minerales. Todo ello, indica que no existiría un efecto permanente de la infraestructura en la actividad económica o en el crecimiento económico de largo plazo (véase el cuadro 3).
Al igual que los modelos en niveles, los modelos en diferencias verifican la presencia de un efecto positivo y significativo de todas las infraestructuras en el crecimiento regional. Asimismo, esta relación prevalece a través de los diversos estimadores utilizados, lo que indica que los resultados son robustos.

Para estos modelos se halla además que el crecimiento del producto per cápita rezagado incide de manera significativa en el crecimiento contemporáneo. No obstante, se encuentra un signo negativo en esta relación. Esto responde a que varias regiones no tienen un crecimiento sostenido, lo que sugiere la necesidad de desarrollar en mayor medida la infraestructura regional para eliminar las trabas al crecimiento económico sostenido. En particular, se debe evitar que el crecimiento económico regional dependa ostensiblemente de proyectos aislados, que se rigen por la volatilidad de los precios en los mercados internacionales.

En relación con estos modelos, la PEA educada sí resultó significativa, lo que muestra la importancia del capital humano para el crecimiento regional de largo plazo. No obstante, dado que el nivel educativo promedio de la población en las regiones es bajo, este factor aún no es tan relevante como podría esperarse.

Con respecto a estos modelos, la importancia relativa de las infraestructuras se invierte y ahora son las telecomunicaciones las que explican en mayor medida los efectos en el crecimiento económico. Ello debido a que las telecomunicaciones están más ligadas a la productividad de los trabajadores (capital humano), que es un determinante del crecimiento de largo plazo regional; el desarrollo de proyectos de electricidad se relacionaría mucho más con los movimientos del producto regional en el corto plazo. En todo caso, tanto la variable de electricidad como la de telecomunicaciones presentan repercusiones en el crecimiento regional similares a las del capital distinto de la infraestructura.

Finalmente, el indicador de carreteras explica una buena parte de las diferencias en el crecimiento regional. Es más, el desarrollo de esta infraestructura resulta más relevante para reducir las brechas del crecimiento de largo plazo entre regiones que para explicar las brechas transitorias en el producto regional. 
CUADRO 3

\section{Modelo en diferencias del crecimiento del PIB per cápita}

\begin{tabular}{|c|c|c|c|c|c|}
\hline Variable & $\begin{array}{c}\text { Pool de datos } \\
\text { (Estimador IV - MGM) }\end{array}$ & $\begin{array}{l}\text { Panel estático } \\
\text { (Estimador MCG) }\end{array}$ & $\begin{array}{c}\text { Panel estático } \\
\text { (Estimador } \\
\text { intragrupos) }\end{array}$ & $\begin{array}{c}\text { Panel } \\
\text { dinámico } \\
\text { (Estimador MGM) }\end{array}$ & $\begin{array}{l}\text { Panel dinámico } \\
\text { (Estimador } \\
\text { MGM en sistema) }\end{array}$ \\
\hline Constante & $\begin{array}{l}-0,0288 * * * \\
(0,0074)\end{array}$ & $\begin{array}{l}-0,0272 * * * \\
(0,0062)\end{array}$ & $\begin{array}{l}-0,0613 * * * \\
(0,0107)\end{array}$ & $\begin{array}{l}-0,0155 \\
(0,0382)\end{array}$ & $\begin{array}{l}-0,0831^{* *} \\
(0,0406)\end{array}$ \\
\hline$\Delta$ Log [PIB per cápita (t-1)] & & & & $\begin{array}{l}-0,1299 * * * \\
(0,0287)\end{array}$ & $\begin{array}{l}-0,1805^{* * *} \\
(0,0574)\end{array}$ \\
\hline$\Delta$ Log [PEA con capital humano] & $\begin{array}{l}0,4666^{* *} \\
(0,2193)\end{array}$ & $\begin{array}{l}0,4097 * * * \\
(0,1542)\end{array}$ & $\begin{array}{l}1,5109 * * * \\
(0,3781)\end{array}$ & $\begin{array}{c}0,3319 \\
(1,2691)\end{array}$ & $\begin{array}{c}2,2662 * \\
(1,3284)\end{array}$ \\
\hline$\Delta$ Log [capital regional] & $\begin{array}{l}0,1050 * * * \\
(0,0324)\end{array}$ & $\begin{array}{l}0,1067 * * * \\
(0,0421)\end{array}$ & $\begin{array}{l}0,1173 * * \\
(0,0444)\end{array}$ & $\begin{array}{r}0,0955^{*} \\
(0,0510)\end{array}$ & $\begin{array}{l}0,1278 * * \\
(0,0560)\end{array}$ \\
\hline$\Delta \log$ [indicador electricidad] & $\begin{array}{l}0,0911 * * * \\
(0,0247)\end{array}$ & $\begin{array}{l}0,0919 * * * \\
(0,0322)\end{array}$ & $\begin{array}{l}0,0997 * * * \\
(0,0341)\end{array}$ & $\begin{array}{l}0,0889 * * * \\
(0,0274)\end{array}$ & $\begin{array}{l}0,1129 * * * \\
(0,0286)\end{array}$ \\
\hline$\Delta \log$ [indicador telecom.] & $\begin{array}{l}0,1209 * * * \\
(0,0220)\end{array}$ & $\begin{array}{l}0,1224 * * * \\
(0,0244)\end{array}$ & $\begin{array}{l}0,1204 * * * \\
(0,0278)\end{array}$ & $\begin{array}{l}0,0831 * * * \\
(0,0307)\end{array}$ & $\begin{array}{l}0,1141 * * * \\
(0,0354)\end{array}$ \\
\hline$\Delta \log$ [indicador transportes] & $\begin{array}{r}0,0463^{*} \\
(0,0275)\end{array}$ & $\begin{array}{l}0,0464 * * * \\
(0,0288)\end{array}$ & $\begin{array}{l}0,0574 * * * \\
(0,0303)\end{array}$ & $\begin{array}{l}0,0921 * * * \\
(0,0168)\end{array}$ & $\begin{array}{l}0,0871 * * * \\
(0,0176)\end{array}$ \\
\hline Número de observaciones & 717 & 717 & 717 & 693 & 695 \\
\hline $\mathrm{R}^{2}$ & 0,0748 & - & - & - & - \\
\hline $\mathrm{R}^{2}$ intragrupos & - & 0,0787 & 0,0862 & - & - \\
\hline $\mathrm{R}^{2}$ entre grupos & - & 0,1190 & 0,1085 & - & - \\
\hline $\mathrm{R}^{2}$ promedio & - & 0,0749 & 0,0609 & - & - \\
\hline Estadístico de Sargan & - & - & - & 1,00 & 1,00 \\
\hline $\begin{array}{l}\text { Arellano-Bond (prueba de } \\
\text { autocorrelación de primer orden) }\end{array}$ & - & - & - & 0,0006 & 0,0009 \\
\hline $\begin{array}{l}\text { Arellano-Bond (prueba de } \\
\text { autocorrelación de segundo orden) }\end{array}$ & - & - & - & 0,4604 & 0,7371 \\
\hline
\end{tabular}

Fuente: elaboración propia sobre la base de estadísticas oficiales de Instituto Nacional de Estadística e Informática (INEI), Organismo Supervisor de Inversión Privada en Telecomunicaciones (OSIPTEL), Ministerio de Energía y Minas (MINEM) y Ministerio de Transportes y Comunicaciones (мTC) e información de Vásquez y Bendezú, Ensayos sobre el rol de la infraestructura vial en el crecimiento económico del Perú, Lima, Consorcio de Investigación Económica y Social (CIES)/Banco Central de Reserva del Perú (BCRP), 2008.

Errores estándar entre paréntesis (robustos según White).

$\mathrm{p}<0,10 ; * * \mathrm{p}<0,05 ; * * * \mathrm{p}<0,01$.

MCG: mínimos cuadrados generalizados.

Estimador de variables instrumentales (IV) a través del método generalizado de momentos (MGM). 


\section{V}

\section{Conclusiones y recomendaciones}

En el presente estudio se pone de manifiesto que las infraestructuras de servicios públicos son relevantes para explicar las brechas en los productos per cápita regionales en el Perú, resultado que está en consonancia no solo con lo planteado por la teoría, sino también en la mayoría de los estudios realizados en numerosos países.

La evidencia respalda la presencia de diferencias significativas en las repercusiones de las distintas infraestructuras - principalmente las de electricidad y telecomunicaciones - en el producto per cápita de cada región, que pueden atribuirse a las brechas en la calidad de la infraestructura regional. Por lo tanto, es necesario que las autoridades de política se enfoquen en el incremento de la cantidad y calidad de las infraestructuras, y en darles el mantenimiento adecuado.

Sin embargo, la evidencia no es contundente con respecto a la incidencia de la infraestructura en el crecimiento económico regional de largo plazo. Existirían otros factores más importantes que explicarían estas diferencias: el capital humano, el progreso tecnológico, la descentralización productiva, entre otros. Por ello, para que la infraestructura repercuta en el largo plazo en el crecimiento regional es necesario realizar políticas complementarias que permitan atenuar las brechas regionales.

La principal implicancia de política del análisis realizado es la necesidad de perseverar en el desarrollo de la infraestructura de servicios públicos. Al respecto, las autoridades deben acelerar los procesos de concesión pendientes y permitir las iniciativas privadas que requieran algún nivel de cofinanciamiento, obviamente en la medida en que los proyectos muestren beneficios económicos y sociales superiores a sus costos. De igual modo, los diferentes niveles de gobierno deben destinar mayores recursos presupuestarios a la ejecución o financiamiento de este tipo de proyectos que previamente hayan sido aprobados por el Sistema Nacional de Inversión Pública.

Una región podrá disfrutar de mayor crecimiento y un nivel de producción per cápita más alto en la medida en que invierta recursos oportuna y eficientemente en el mejoramiento de su infraestructura de carreteras, telecomunicaciones y electricidad. En este sentido, resulta injustificable que exista en el Perú un grupo de gobiernos regionales y municipales con un muy bajo nivel de ejecución presupuestal, principalmente en infraestructura, y que muchos de ellos se encuentren precisamente en las zonas más deprimidas del país.

Hay que reconocer, sin embargo, que los resultados numéricos deben tomarse con cautela debido a los problemas de calidad de la información a nivel regional. Por ello, se sugiere dedicar mayores esfuerzos a la revisión de la información actualmente disponible, así como a la producción y procesamiento de la información que se ha identificado como necesaria para obtener resultados más confiables. He aquí, por lo tanto, una tarea adicional para las autoridades, más precisamente para las propias instituciones que producen y manejan estas estadísticas, aunque también deberían comprometer su apoyo las universidades y centros de investigación como potenciales usuarios de la información. 
ANEXO

Estadísticas de las variables utilizadas

\begin{tabular}{|c|c|c|c|c|c|c|}
\hline Variable & Variabilidad & Media & $\begin{array}{l}\text { Desviación } \\
\text { estándar }\end{array}$ & Mínimo & Máximo & $\begin{array}{c}\text { Número de } \\
\text { observaciones }\end{array}$ \\
\hline PIB per cápita ${ }^{\mathrm{a}}$ & $\begin{array}{l}\text { Promedio } \\
\text { Entre grupos } \\
\text { Intragrupos }\end{array}$ & 3907,8 & $\begin{array}{l}2455,9 \\
2229,4 \\
1108,8\end{array}$ & $\begin{array}{r}1216,2 \\
1638,0 \\
-4639,0\end{array}$ & $\begin{array}{r}16133,3 \\
10447,8 \\
9593,3\end{array}$ & 928 \\
\hline PEA per cápita & $\begin{array}{l}\text { Promedio } \\
\text { Entre grupos } \\
\text { Intragrupos }\end{array}$ & 0312,0 & $\begin{array}{l}0043,5 \\
0034,6 \\
0027,1\end{array}$ & $\begin{array}{l}0230,7 \\
0255,8 \\
0221,4\end{array}$ & $\begin{array}{l}0478,3 \\
0407,2 \\
0443,7\end{array}$ & 903 \\
\hline $\begin{array}{l}\text { PEA con educación secundaria o } \\
\text { superior per cápita }^{\mathrm{a}}\end{array}$ & $\begin{array}{l}\text { Promedio } \\
\text { Entre grupos } \\
\text { Intragrupos }\end{array}$ & 0154,6 & $\begin{array}{ll}0 & 075,6 \\
0 & 059,4 \\
0 & 048,3\end{array}$ & $\begin{array}{l}0028,3 \\
0073,7 \\
0057,3\end{array}$ & $\begin{array}{l}0398,3 \\
0295,9 \\
0354,1\end{array}$ & 890 \\
\hline $\begin{array}{l}\text { Capital regional distinto de } \\
\text { infraestructura per cápita }\end{array}$ & $\begin{array}{l}\text { Promedio } \\
\text { Entre grupos } \\
\text { Intragrupos }\end{array}$ & 0000,3 & $\begin{array}{l}0000,6 \\
0000,6 \\
0000,1\end{array}$ & $\begin{array}{r}0000,1 \\
0000,0 \\
-0000,9\end{array}$ & $\begin{array}{l}0002,9 \\
0002,3 \\
0001,0\end{array}$ & 812 \\
\hline Electricidad per cápitab & $\begin{array}{l}\text { Promedio } \\
\text { Entre grupos } \\
\text { Intragrupos }\end{array}$ & 0090,2 & $\begin{array}{ll}0 & 199,1 \\
0 & 054,1 \\
0 & 191,8\end{array}$ & $\begin{array}{r}0000,2 \\
0013,5 \\
-0103,3\end{array}$ & $\begin{array}{l}1393,2 \\
0237,4 \\
1246,0\end{array}$ & 823 \\
\hline Telecomunicaciones per cápitac & $\begin{array}{l}\text { Promedio } \\
\text { Entre grupos } \\
\text { Intragrupos }\end{array}$ & 0002,4 & $\begin{array}{l}0007,9 \\
0004,6 \\
0006,5\end{array}$ & $\begin{array}{r}0000,0 \\
0000,1 \\
-0018,7\end{array}$ & $\begin{array}{ll}0 & 119,8 \\
0 & 023,1 \\
0 & 099,1\end{array}$ & 888 \\
\hline Transportes ${ }^{d}$ & $\begin{array}{l}\text { Promedio } \\
\text { Entre grupos } \\
\text { Intragrupos }\end{array}$ & 0432,9 & $\begin{array}{l}0558,6 \\
0525,9 \\
0215,3\end{array}$ & $\begin{array}{r}0000,7 \\
0001,3 \\
-1007,9\end{array}$ & $\begin{array}{l}3977,8 \\
2640,3 \\
1770,4\end{array}$ & 742 \\
\hline
\end{tabular}

Fuente: elaboración propia sobre la base de estadísticas oficiales de Instituto Nacional de Estadística e Informática (INEI), Organismo Supervisor de Inversión Privada en Telecomunicaciones (OSIPTEL), Ministerio de Energía y Minas (MINEM) y Ministerio de Transportes y Comunicaciones (MTC) e información de Vásquez y Bendezú, Ensayos sobre el rol de la infraestructura vial en el crecimiento económico del Perú, Lima, Consorcio de Investigación Económica y Social (CIES)/Banco Central de Reserva del Perú (BCRP), 2008.

PIB: producto interno bruto. PEA: población económicamente activa.

a Soles de 1994

b Megavatios.

c Líneas fijas y móviles.

d Indicador de calidad entre acervo de redes viales.

Alesina, A. y R. Perotti (1993), "Income distribution, political instability, and investment", NBER Working Paper, $\mathrm{N}^{\circ}$ 4486, Cambridge, Massachusetts, National Bureau of Economic Research.

Arellano, M. y S. Bond (1991), "Some tests of specification for panel data: Monte Carlos evidence and an application to employment equations", Review of Economic Studies, vol. 58, No 2, Wiley Blackwell.

Arellano, M. y O. Bover (1995), "Another look at the instrumental variable estimation of error-component models", Journal of Econometrics, vol. 68, $\mathrm{N}^{\circ} 1$, Amsterdam, Elsevier.

Aschauer, D. (1989), "Is public expenditure productive?", Journal of Monetary Economics, vol. 23, No 2, Amsterdam, Elsevier.

Bandyopadhyay, S. y S. Devarajan (1993), "Using project rates of return to inform sector allocation decisions", Washington, D.C., Banco Mundial, inédito.

Barro, R. y X. Sala-i-Martin (1990), "Public finance in models of economic growth", NBER Working Paper, $\mathrm{N}^{\circ} 3362$, Cambridge, Massachusetts, National Bureau of Economic Research.

Blundell, R. y S. Bond (1998), "Initial conditions and moment restrictions in dynamic panel data models", Journal of Econometrics, vol. 87, $\mathrm{N}^{\circ}$ 1, Amsterdam, Elsevier.
Calderón, C. y L. Servén (2004a), "Trends in infrastructure in Latin America, 1980-2001”, Documentos de trabajo, № 269, Santiago de Chile, Banco Central de Chile, septiembre.

(2004b), "The effects of infrastructure development on growth and income distribution", Documentos de trabajo, $\mathrm{N}^{\circ}$ 270, Santiago de Chile, Banco Central de Chile, septiembre.

Cameron, C. y P. Trivedi (2009), Microeconometrics Using Stata, Texas, Stata Press.

Canning, D. (1999), "Infrastructure's contribution to aggregate output", Policy Research Working Paper, No 2246, Washington, D.C., Banco Mundial.

Canning, D. y E. Bennathan (2000), "The social rate of return on infrastructure investments", Policy Research Working Paper, No 2390, Washington, D.C., Banco Mundial.

Canning, D. y P. Pedroni (2004), "The effect of infrastructure on long run economic growth", noviembre, inédito.

Cárdenas, M., A. Gaviria y M. Meléndez (2005), "La infraestructura de transporte en Colombia", agosto, inédito.

Davis, D. (1998), "The home market, trade and industrial structure", American Economic Review, vol. 88, No 5, Nashville, Tennessee, American Economic Association, diciembre. 
Devarajan, S., V. Swaroop y H. Zou (1996), "The composition of public expenditure and economic growth", Journal of Monetary Economics, vol. 37, $\mathrm{N}^{\mathrm{o}}$ 2-3, Amsterdam, Elsevier.

Dolado, J., T. Jenkinson y S. Sosvilla-Rivero (1990), "Cointegration and unit root tests", Journal of Economic Surveys, vol. 4, Nº 3, Wiley.

Duggal, V., C. Saltzman y L. Klein (1999), "Infrastructure and productivity: a nonlinear approach", Journal of Econometrics, vol. 92, $\mathrm{N}^{\mathrm{o}}$ 1, Amsterdam, Elsevier.

Easterly, W. y S. Rebelo (1993), "Fiscal policy and economic growth: An empirical investigation", Journal of Monetary Economics, vol. $32, \mathrm{~N}^{\circ} 4$, Amsterdam, Elsevier.

Esfahani, H. y M. Ramírez (2003), "Institutions, infrastructure and economic growth", Journal of Development Economics, vol. 70, $\mathrm{N}^{\mathrm{o}} 2$, Amsterdam, Elsevier.

Estache, A. y M. Fay (2007), "Current debates on infrastructure policy", Policy Research Working Paper, No 4410, Washington, D.C., Banco Mundial, noviembre.

Fay, M. y M. Morrison (2005), Infraestructura en América Latina y el Caribe: Tendencias recientes y retos principales, Washington, D.C., Banco Mundial.

García-Milà, T., T. McGuire y R. Porter (1996), "The effect of public capital in state-level production functions reconsidered", Review of Economics and Statistics, vol. 78, № 1, Cambridge, Massachusetts, The міт Press.

Glomm, G. y B. Ravikumar (1994), "Public investment in infrastructure in a simple growth model", Journal of Economic Dynamics and Control, vol. 18, $\mathrm{N}^{\circ}$ 6, Amsterdam, Elsevier.

González, J., J. Guasch y T. Serebrisky (2007), "Latin America: Addressing high logistics costs and poor infrastructure for merchandise transportation and trade facilitation", Consulta de San José 2007, Banco Mundial, agosto.

Gramlich, E. (1994), "Infrastructure investment: A review essay", Journal of Economic Literature, vol. 32, N ${ }^{\circ} 3$, Nashville, Tennessee, American Economic Association.

Guasch, J., J. Laffont y S. Straub (2005), "Infrastructure concessions in Latin America: government-led renegotiations", Policy Research Working Paper, $\mathrm{N}^{\circ}$ 3749, Washington, D.C., Banco Mundial.

Holtz-Eakin, D. (1994), "Public-sector capital and the productivity puzzle", Review of Economics and Statistics, vol. 76, № 1 , Cambridge, Massachusetts, The MIT Press.

Hulten, C. (1996), "Infrastructure capital and economic growth: How well you use it may be more important than how much you have", NBER Working Paper, $\mathrm{N}^{\circ}$ 5847, Cambridge, Massachusetts, National Bureau of Economic Research.

Hulten, C., E. Bennathan y S. Srinivasan (2006), "Infrastructure, externalities, and economic development", World Bank Economic Review, vol. 20, $\mathrm{N}^{\mathrm{o}}$ 2, Washington, D.C., Banco Mundial.

Im, K., M.H. Pesaran y Y. Shin (2003), "Testing for unit roots in heterogeneous panels", Journal of Econometrics, vol. 115, $\mathrm{N}^{\mathrm{o}} 1$, Amsterdam, Elsevier.

Laffont, J. y J. Tirole (1993), A Theory of Incentives in Procurement and Regulation, Cambridge, Massachusetts, The MIT Press.

Levin, A., Ch. Lin y Ch. Chu (2002), "Unit root tests in panel data: Asymptotic and finite-sample properties", Journal of Political Economy, vol. 108, № 1, Amsterdam, Elsevier.

Limao, N. y A. Venables (2001), "Infrastructure, geographical disadvantage, transport costs and trade", World Bank Economic Review, vol. 15, $\mathrm{N}^{\mathrm{o}}$ 3, Washington, D.C., Banco Mundial.
Limi, A. (2008), "Effects of improving infrastructure quality on business costs: Evidence from firm-level data", Policy Research Working Paper, $\mathrm{N}^{\circ}$ 4581, Washington, D.C., Banco Mundial, marzo.

Loayza, N., P. Fajnzylber y C. Calderón (2002), Economic Growth in Latin America and the Caribbean: Stylized Facts, Explanations, and Forecasts, Washington, D.C., Banco Mundial, junio.

Mankiw, G., D. Romer y D. Weil (1992), "A contribution to the empirics of economic growth", The Quarterly Journal of Economics, vol. 107, No 2, Cambridge, Massachusetts, The MIT Press, mayo.

Mia, I., J. Estrada y T. Geiger (2007), Benchmarking National Attractiveness for Private Investment in Latin American Infrastructure, Foro Económico Mundial.

Munnell, A. (1990), "Why has productivity growth declined? Productivity and public investment", New England Economic Review, Boston, Banco de la Reserva Federal de Boston, enero-febrero.

Nadiri, M. Ishaq y T. Mamuneas (1994), "Infrastructure and public R\&D investments, and the growth of factor productivity in US manufacturing industries", NBER Working Paper, $N^{\circ} 4845$, Cambridge, Massachusetts, National Bureau of Economic Research, agosto.

Noriega, A. y M. Fontela (2005), "Public infrastructure and economic growth in Mexico", Universidad de Guanajato/Centro de Investigación y Docencia Económicas (CIDE), abril, inédito.

Pritchett, L. (1996), "Mind your P's and Q's. The cost of public investment is not the value of public capital", Policy Research Working Paper, $\mathrm{N}^{\circ}$ 1660, Washington, D.C., Banco Mundial.

Prud'homme, R. (2005), "Infrastructure and development", Annual World Bank Conference on Development Economics, 2005. Lessons of Experience, F. Bourguignon y B. Pleskovic (eds.), Washington, D.C., Banco Mundial/Oxford University Press.

Rivera, J. y P. Toledo (2004), "Efectos de la infraestructura pública sobre el crecimiento de la economía, evidencia para Chile", Estudios de economía, vol. 31, Santiago de Chile, Universidad de Chile.

Romp, W. y J. de Haan (2007), "Public capital and economic growth: A critical survey", Perspektiven der Wirtschaftspolitik, vol. 8, Blackwell Publishing.

Sánchez-Robles, B. (1998), "Infrastructure investment and growth: Some empirical evidence", Contemporary Economic Policy, vol. 16, № 1 , Fountain Valley, Western Economic Association International.

Solow, R. (1956), "A contribution to the theory of economic growth", Quarterly Journal of Economics, vol. 70, № 1, Oxford, Oxford University Press.

Straub, S. (2008a), "Infrastructure and growth in developing countries: Recent advances and research challenges", Policy Research Working Paper, $\mathrm{N}^{\circ} 4460$, Washington, D.C., Banco Mundial, enero. (2008b), "Infrastructure and development: A critical appraisal of the macro level literature", Policy Research Working Paper, $\mathrm{N}^{\mathrm{o}} 4590$, Washington, D.C., Banco Mundial, abril.

Straub, S., C. Vellutini y M. Warlters (2008), "Infrastructure and economic growth in East Asia", Policy Research Working Paper, $\mathrm{N}^{\circ}$ 4589, Washington, D.C., Banco Mundial, abril.

Urrunaga, R. (2010), "Relevancia de la infraestructura y análisis de los sobrecostos que genera su déficit", Apuntes, $\mathrm{N}^{\circ} 65$, Lima, Universidad del Pacífico, enero.

Vásquez, A. y L. Bendezú (2008), Ensayos sobre el rol de la infraestructura vial en el crecimiento económico del Perú, Lima, Consorcio de Investigación Económica y Social (CIES)/ Banco Central de Reserva del Perú (BCRP). 